\title{
Comparison of structured- and unstructured-grid, compressible and incompressible methods using the vortex pairing problem.
}

Panagiotis Tsoutsanis, Ioannis W. Kokkinakis, László Könözsy, Dimitris Drikakis

Fluid Mechanics and Computational Science, Cranfield University, Cranfield, MK43 OAL, United Kingdom

Robin J.R. Williams, David L. Youngs

AWE, Aldermaston, Reading RG7 4PR, United Kingdom

Corresponding author: Prof Dimitris Drikakis, Fluid Mechanics and Computational Science Centre,

Cranfield University, Cranfield, MK43 0AL, United Kingdom, d.drikakis@cranfield.ac.uk, Tel: +44-1234-754796 


\begin{abstract}
The accuracy, robustness, dissipation characteristics and efficiency of several structured and unstructured grid methods are investigated with reference to the low Mach double vortex pairing flow problem. The aim of the study is to shed light into the numerical advantages and disadvantages of different numerical discretizations, principally designed for shock-capturing, in low Mach vortical flows. The methods include structured and unstructured finite volume and Lagrange-Remap methods, with accuracy ranging from $2^{\text {nd }}$ to $9^{\text {th }}$-order, with and without applying low-Mach corrections. Comparison of the schemes is presented for the vortex evolution, momentum thickness, as well as for their numerical dissipation versus the viscous and total dissipation. The study shows that the momentum thickness and large scale features of a basic vortical structure are well resolved even at the lowest grid resolution of $32 \times$ 32 provided that the numerical schemes are of a high-order of accuracy or the numerical framework is sufficiently non-dissipative. The implementation of the finite volume methods in unstructured triangular meshes provides the best results even without low Mach number corrections provided that a higher-order advective discretization for the advective fluxes is employed. The compressible Lagrange-Remap framework is computationally the fastest one, although the numerical error for the momentum thickness does not reduce as fast as for other numerical schemes and computational frameworks, e.g. , when higher-order schemes are utilized. It is also shown that the low-Mach number correction has a lesser effect on the results as the order of the spatial accuracy increases.
\end{abstract}

\title{
1. Introduction
}

The presence of a wide-range of spatial and temporal scales in complex flows featuring vorticity dynamics prohibits the use of direct numerical simulations to resolve all of the scales within a flow, even with today's computing power, and it is expected that it will remain the case in the foreseeable future. Therefore, in many practical applications the simulations still remain under-resolved, such that the large scales are resolved and the smallest scales are modeled.

It is well known that high-order schemes are superior to low-order ones both in terms of accuracy and computational efficiency. However, high-order schemes usually lack the robustness of their low-order counterparts. Furthermore, it is not yet well understood how different unstructured and structured-grid based computational frameworks and numerical discretisation schemes influence the accuracy (and efficiency) of simulations in vortical flows. The primary motivation of this work is to address the behavior of different high-resolution and high-order numerical schemes and computational frameworks in a basic (prototypical) example of vortical flow. Although the present study does 
not concern an assessment of the accuracy of numerical methods in turbulent flows, the methods employed here are widely used in implicit large eddy simulations (ILES) [1-4], which rely on the non-linear numerical dissipation of high-resolution schemes to locally and dynamically produce similar effects achieved by explicit subgrid models used in classical LES. Since the accuracy of numerical schemes used in ILES is sensitive to the critical balance of the dissipation and dispersion contributions to the numerical solution [5-9], which strongly depend on the design details of each high-resolution non-oscillatory finite volume method, it is of paramount importance to understand the performance of high-order schemes in prototypical vortical flows, especially when using under-resolved grid arrangements. Therefore, the numerical accuracy issues for the double vortex pairing (DVP) problem are also pertinent to more complex flows.

Four different numerical frameworks are used in this study. These are an incompressible structured-grid finite volume, a compressible structured-grid finite volume, a compressible unstructured-grid finite volume, and a compressible Lagrange-Remap framework. Determining which of these frameworks is "the best" is not the scope of the study, as it is appreciated that each of these frameworks may exhibit different behavior depending on the types of flow problems encountered. On the other hand, it is essential to understand the underlying characteristics of each of these numerical frameworks when used in conjunction with high-resolution/high-order schemes at coarse grid resolutions and for low Mach number flows such as the basic double vortex pairing problem.

The test problem is related to the experimental work carried out by Winant and Browand [10], who investigated the vortex formation in a shear layer and the subsequent double vortex interaction and pairing process into a single vortex. A mixing layer is formed by bringing two streams of water, moving at different velocities, together in a lucitewalled channel. In the experiments conducted [10] it was observed that unstable waves grow downstream and the fluid subsequently rolls up into discrete two-dimensional vortical structures. These turbulent-like vortices interact by rolling around each other, ultimately forming a single vortical structure with approximately twice the spacing of the former vortices as illustrated in Figure 1. This pairing process is observed to occur repeatedly, controlling the growth of the mixing layer.

The flow conditions (stream velocities, length-scales, Reynolds number etc.) investigated in the numerical simulations are taken from the aforementioned experiment with the aim of calculating the evolution of some of the observed large-scale structures. Simulation of vortical flows requires modeling as wide a range of structure sizes as possible (i.e. maximizing the achievable dynamic range). Hence, one method of testing the suitability of the numerics is to ask the question: "What is the lowest resolution that can be used to model a basic vortical structure?"

The objective is to demonstrate the capability of compressible and incompressible high-resolution methods to obtain accurate results at coarse-grid resolutions $\left(32^{2}\right.$ and $\left.64^{2}\right)$, particularly for near incompressible flows. Even 


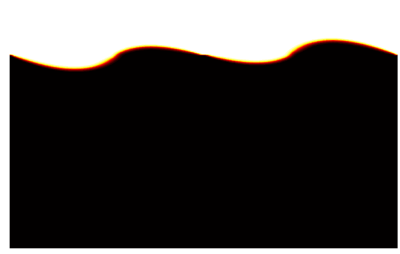

(a) $1.0 \mathrm{~s}$

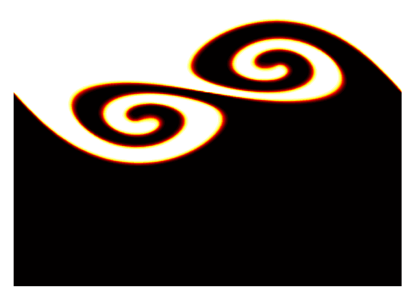

(d) $4.0 \mathrm{~s}$

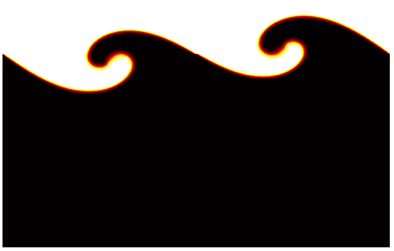

(b) $2.0 \mathrm{~s}$

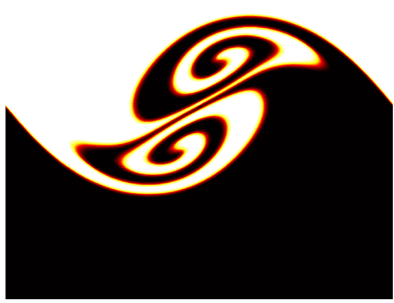

(e) $5.0 \mathrm{~s}$

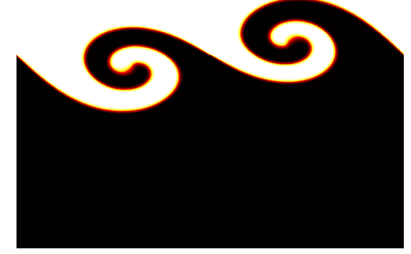

(c) $3.0 \mathrm{~s}$

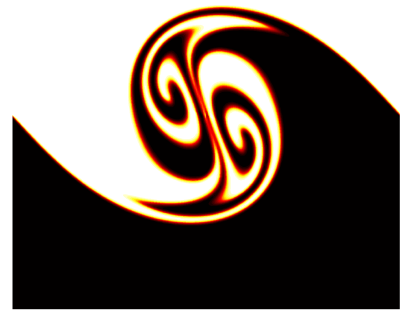

(f) $6.0 \mathrm{~s}$

Figure 1: Time evolution of the double vortex pairing process on a $256^{2}$ grid cell resolution using the WENO $9^{\text {th }}$ order scheme in the compressible structured-grid framework; passive scalar contours $(\mathrm{c}=0.25, \mathrm{c}=0.5, \mathrm{c}=0.75)$.

in highly compressible flows, small scale vortical structures are usually low Mach number features. Hence the test problem is of relevance to a range of compressible and incompressible flows. The numerical method survey conducted consists of a structured-grid finite-volume compressible solver using high-order reconstruction methods with 1D swept directional stencils; a compressible unstructured-grid solver, also using high order reconstruction methods, but allowing for multidimensional stencils; a compressible Lagrange-remap solver (LR); and an incompressible finite volume structured-grid solver in order to include a reference high-resolution incompressible solution for the present low speed problem. Viscosity is included in the test problem and the highest resolution simulations $\left(256^{2}\right)$ fully resolves the velocity field.

The paper is organized as follows. First, the initial and boundary conditions of the double vortex pairing are described in $\S 2$. Then, in $\S 3$, a description of the numerical methods and frameworks employed in this study are presented, followed by a presentation in $\S 4$ of all the statistical quantities used in assessing the accuracy of the schemes. The results obtained by the various schemes and frameworks are categorized in terms of vortex evolution, momentum thickness, (numerical) kinetic energy dissipation and computational efficiency in subsections §5.1, 5.2, 5.3 and 5.4, respectively. Finally in $\S 6$ conclusions from the present study are drawn. 


\section{Double Vortex Problem Description}

\subsection{Initial and Boundary Conditions}

The initial conditions described in [10] consist of two co-flowing velocities, $u_{1}=4.06 \mathrm{~cm} / \mathrm{s}$ (lower stream) and $u_{2}=1.44 \mathrm{~cm} / \mathrm{s}$ (upper stream). The mixing layer comprises of a single component, single phase fluid. The calculation is performed in a frame of reference moving with the mean stream velocity, $U=2.75 \mathrm{~cm} / \mathrm{s}$, and focuses on the evolution of the large scale vortex from the two original smaller vortices. The final structure has a wavelength of $L \approx 6 \mathrm{~cm}$, which is the length of the edges of the computational box used to contain the double vortex evolution and subsequent merger (Fig. 1).

The two streams have a velocity difference of $\Delta U=2.62 \mathrm{~cm} / \mathrm{s}$ and same physical properties such as density and viscosity. The computations performed maintain the velocity difference $\Delta U=2.62 \mathrm{~cm} / \mathrm{s}$ but assign equal and opposing free-stream velocities to the two layers similar to other numerical double vortex pairing investigations [11]. Thus the free-stream properties for velocity are $U_{\infty}^{\text {Lower }}=\Delta U / 2=1.31 \mathrm{~cm} / \mathrm{s}$ and $U_{\infty}^{\text {Upper }}=-U_{\infty}^{\text {Lower }}$, density $\rho_{\infty}=1 \mathrm{gr} / \mathrm{cm}^{3}$ and kinematic viscosity $v_{\infty}=0.01 \mathrm{~cm}^{2} / \mathrm{s}$. The Mach number based on the relative velocity of the two streams $(\Delta U)$ is equal to 0.2 , or otherwise based on the free-stream velocity (the so-called convective Mach number) equal to 0.1. In either case the free-stream speed of sound is given by $\alpha_{\infty}=\sqrt{\gamma P_{\infty} / \rho_{\infty}}$. Finally, the adiabatic index $\gamma$ is equal to $5 / 3$ for an ideal monatomic gas and the free-stream pressure is $P_{\infty}=10.3 \mathrm{~N} / \mathrm{m}^{2}$ and assumed constant in the domain. Note that the Mach number can also be lowered by simply increasing $P_{\infty}$.

The unperturbed streamwise velocity profile is given by equation:

$$
u=-\frac{1}{2} \Delta U \tanh \left(\frac{y}{2 \theta_{0}}\right)
$$

where $\theta_{0}$ is the initial momentum thickness equal to $0.03 \mathrm{~cm}$.

The Reynolds number based on $\Delta U, L$ and $v$ is $\operatorname{Re}=(\Delta U) L / v=1600$. A stream function $(\psi)$ is used to add a divergence-free initial perturbation to both velocity components $\left(u^{\prime}, v^{\prime}\right)$. The fluctuations are calculated as:

$$
u^{\prime}=-\frac{\partial \psi}{\partial y}, \quad v^{\prime}=\frac{\partial \psi}{\partial x}
$$

The stream function $\psi$ is the sum of two Kelvin-Helmholtz instability eigenmodes given by [12]:

$$
\psi=A_{1}(y) \frac{v_{1}}{k_{1}} \cos \left(k_{1} x\right) \exp \left(-k_{1}|y|\right)+A_{2}(y) \frac{v_{2}}{k_{2}} \cos \left(k_{2} x\right) \exp \left(-k_{2}|y|\right)
$$


with the two corresponding wavenumbers, $k_{1}$ and $k_{2}$, set equal to:

$$
k_{1}=\frac{2 \pi}{L}, \quad k_{2}=\frac{4 \pi}{L}
$$

and

$$
A_{i}=\frac{1-\exp \left[-2 k_{i}\left(\frac{L}{2}-|y|\right)\right]}{1-\exp \left(-k_{i} L\right)}
$$

The two velocity amplitudes are $v_{1}=0.025 \Delta U$ and $v_{2}=0.05 \Delta U$. Similar to previous numerical studies [11], the boundary conditions are periodic in the $\mathrm{x}$-direction (streamwise) and reflective $(\partial v / \partial y=0)$ in the $\mathrm{y}$-direction. The reflective condition in the $y$-direction is required to avoid viscouss dissipation at the boundaries.

\section{Description of methods}

\subsection{Compressible methods}

The 2D viscous compressible Navier-Stokes with heat conduction are considered in the following form:

$$
\frac{\partial}{\partial t} \mathbf{U}+\frac{\partial}{\partial x}\left(\mathbf{F}_{\mathbf{u}}-\mathbf{G}_{\mathbf{u}}\right)+\frac{\partial}{\partial y}\left(\mathbf{F}_{\mathbf{v}}-\mathbf{G}_{\mathbf{v}}\right)=0
$$

where $\mathbf{U}$ is the vector of the conserved variables, $\mathbf{F}_{\mathbf{u}}, \mathbf{G}_{\mathbf{u}}$, and $\mathbf{F}_{\mathbf{v}}, \mathbf{G}_{\mathbf{v}}$ are the convective and viscous flux vectors in $x, y$ Cartesian coordinates directions respectively, given by:

$$
\begin{gathered}
\mathbf{U}=\left[\begin{array}{c}
\rho \\
\rho u \\
\rho v \\
E \\
\rho \varphi
\end{array}\right], \mathbf{F}_{\mathbf{u}}=\left[\begin{array}{c}
\rho u \\
\rho u^{2}+p \\
\rho u v \\
u(E+p) \\
\rho u \varphi
\end{array}\right], \mathbf{F}_{\mathbf{v}}=\left[\begin{array}{c}
\rho v \\
\rho v u \\
\rho v^{2}+p \\
v(E+p) \\
0 \\
\tau_{x x} \\
\tau_{x y} \\
\theta_{x} \\
0
\end{array}\right], \quad \mathbf{G}_{\mathbf{v}}=\left[\begin{array}{c}
\rho v \varphi \\
\tau_{y y} \\
\tau_{y x} \\
\theta_{y} \\
0
\end{array}\right]
\end{gathered}
$$


where $\rho$ is the density, $u$ and $v$ are the velocity components in the $x$ and $y$ directions respectively, $p$ represents static pressure, $E=p /(\gamma-1)+0.5 \rho\left(u^{2}+v^{2}\right)$ is the total energy per unit volume, $\varphi$ is a passive scalar, $\gamma$ is the ratio of specific heats, $\tau_{i j}$ the stresses tensor, and $\theta$ is defined by the following relations:

$$
\begin{aligned}
& \theta_{x}=u \tau_{x x}+v \tau_{x y}+\frac{\mu \gamma}{\operatorname{Pr}(\gamma-1)} \frac{\partial T}{\partial x} \\
& \theta_{y}=u \tau_{y x}+v \tau_{y y}+\frac{\mu \gamma}{\operatorname{Pr}(\gamma-1)} \frac{\partial T}{\partial y}
\end{aligned}
$$

The ideal gas law is used with $\gamma=5 / 3, T=p / \rho$ is the temperature, $\operatorname{Pr}$ is the Prandtl number and $\mu$ is the dynamic viscosity; the initial conditions and fluid parameters were previously defined in §2.1.

The spatial domain is discretized by conforming elements. Its cell element $i$ of volume $V_{i}$ (area for 2D), can be of triangular or quadrilateral shape as shown in Figure 3. Integrating equation (6) over a mesh element, leads to the following semi-discrete finite-volume formulation:

$$
\frac{d}{d t} \mathbf{U}_{i}+\frac{1}{V_{i}} \oint_{\partial V_{i}}\left(\mathbf{F}_{n}^{\mathbf{c}}-\mathbf{F}_{n}^{\mathbf{v}}\right) d A=0
$$

where $A$ is the length of the corresponding side, and $\mathbf{F}_{n}^{\mathbf{c}}-\mathbf{F}_{n}^{\mathbf{v}}$ are the projections of the convective and viscous flux vectors normal to the sides given by:

$$
\mathbf{F}_{n}^{\mathbf{c}}=\left[\begin{array}{c}
\rho \mathrm{V}_{n} \\
\rho u \mathrm{~V}_{n}+n_{x} p \\
\rho v \mathrm{~V}_{n}+n_{y} p \\
\mathrm{~V}_{n}(E+p)
\end{array}\right], \quad \mathbf{F}_{n}^{\mathbf{v}}=\left[\begin{array}{c}
0 \\
n_{x} \tau_{x x}+n_{y} \tau_{x y} \\
n_{x} \tau_{y x}+n_{y} \tau_{y y} \\
n_{x} \theta_{x}+n_{y} \theta_{y}
\end{array}\right]
$$

$\mathbf{U}_{i}(t)$ is the conserved vector at time level $t$, and $\mathrm{V}_{n}$ is the velocity normal to the side $A$ defined by $\mathrm{V}_{n}=n_{x} u+n_{y} v$. Assuming that the element consists of $L$ sides and denoting by $\mathbf{n}_{j}$ the outward unit vector for side $A_{j}$, then the integral over the element boundary $\partial V_{i}$ splits into the sum of integrals over each side resulting in the following expression:

$$
\frac{d}{d t} \mathbf{U}_{i}=\mathbf{R}_{i}
$$

with

$$
\mathbf{R}_{i}=-\frac{1}{V_{i}} \sum_{j=1}^{L} \int_{A_{j}} \mathbf{F}_{n, j}^{\mathbf{c}} d A+\frac{1}{V_{i}} \sum_{j=1}^{L} \int_{A_{j}} \mathbf{F}_{n, j}^{\mathbf{v}} d A
$$




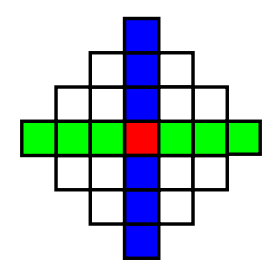

Figure 2: Structured solver reconstruction stencils (1D-split) for a $5^{\text {th }}$ order scheme.

A passive scalar field $(\varphi)$ is used to visualize the formation process of the vortices and merger. The passive scalar is advected according to the resolved viscous velocity field and is therefore given by:

$$
\frac{d \rho \varphi}{d t}+\frac{1}{V_{i}} \oint_{\partial V_{i}} \mathrm{~V}_{n} \rho \varphi d A=0
$$

In the case of the incompressible structured-grid framework, the solution is advanced in time using a dual-time stepping method with inner pseudo-time steps and the passive scalar is therefore given by:

$$
\frac{\partial \varphi}{\partial \tau}=-\frac{\partial \varphi}{\partial t}-(\mathbf{u} \cdot \nabla) \varphi
$$

\subsubsection{Compressible structured-grid framework}

The structured-grid code solves the full Navier-Stokes equations using a finite volume Godunov-type [13, 14] method. The intercell numerical fluxes are computed based on the solution to the Riemann problem using the reconstructed variables at the left and right (or upper and lower) cell interfaces. The reconstruction stencil is a onedimensional swept directional stencil, as illustrated in Figure 2.

The Riemann problem is solved using the Harten, Lax, van Leer, and (the missing) "Contact" (HLLC) approximate Riemann solver [15]. The reconstructed values utilized in the HLLC Riemann solver are obtained primarily by two different limiter approaches, the Monotone Upstream-centered Schemes for Conservation Laws (MUSCL) [16] and the Weighted-Essentially-Non-Oscillatory (WENO) reconstruction methods [17]. For each reconstruction technique a variety of differing orders of accuracy are examined, all of odd number. MUSCL is employed using $3^{\text {rd }}$ and $5^{\text {th }}$ order of accuracy schemes [18, 19] (henceforth labeled in the figures as M3 and M5, respectively), whereas WENO uses the $5^{\text {th }}$ and $9^{\text {th }}$ order of accuracy schemes $[20,21]$ in conjunction with the relative smoothness limiter of [22] (henceforth labeled as W5 and W9, respectively), which are extensions of the original WENO scheme [17].

All the reconstruction techniques used in this paper have been further augmented with a low-Mach limiting scheme [23], which involves an additional stage in the reconstruction process for the velocity vector. This low-Mach number correction (labeled LM) ensures uniform dissipation of kinetic energy in the limit of zero Mach number, thus extending 
the validity of Godunov type method to at least Mach $\approx 10^{-4}$ via a progressive central differencing of the velocity components. The viscous part of the equations is solved using a second order central difference scheme. Finally, the solution is advanced in time using a three-stage total variation diminishing (TVD) Runge-Kutta (RK) method $[14,15,24]$.

\subsubsection{Compressible unstructured-grid framework}

The numerical approach of $[25,26]$ is adopted in the present study which is suitable for unstructured meshes with various types of element shapes in $2 \mathrm{D}$ and $3 \mathrm{D}$, where it has been previously used successfully for laminar, transitional and turbulent flows [27, 28]. A Gaussian numerical quadrature of appropriate order for the order of the polynomial used is implemented for the approximation of the integral expressions of the fluxes.

The calculation of the numerical convective and viscous fluxes requires the knowledge of the pointwise values of the conserved vector as well as the velocity and temperature gradients at each Gaussian integration point. These pointwise values are approximated through an interpolation (reconstruction) procedure of a desired order of accuracy utilizing the cell averages. The latter requires a recursive stencil construction process where the direct side neighbor elements are added until a target number $M$ of stencil elements has been reached. For MUSCL types of schemes only one central stencil is used, however for WENO schemes, in addition to the central stencil, several additional directional stencils are also used as shown in Figure 3.

The reconstruction is carried out in a transformed system of co-ordinates in order to minimize scaling effects that appear in stencils consisting of elements of different sizes, as well as to improve the condition number of the system of equations [25, 26]. For computing the degrees of freedom, a minimum of $K$ cells are needed in the stencil in addition to the target cell. Using the minimum possible number of cells in the stencil $(M \equiv K)$ has been found to produce ill-conditioned systems [26, 29-31], hence the choice to use $M=2 K$ improves the robustness of the method. This is especially worthwhile since no substantial performance penalty is incurred as a result of this improvement [26, 31, 32]. The resulting least-squares system is solved by a QR decomposition and the reconstruction polynomial is computed.

In the present study two types of schemes are employed for the convective part, a MUSCL type of scheme using the TVD-type slope limiter of Barth and Jespersen [33] and the WENO implementation of [25, 26] carried out in characteristic variables, both of them satisfying the $k$-exactness criteria. For the viscous part a linear reconstruction polynomial of the same order for the velocity and temperature field is constructed using the same central stencil as for the conserved vector. The discontinuous states of the convective fluxes are approximated by the HLLC Riemann solver of Toro [15], and the central averaging approach is used for the discontinuous viscous flux. The solution is advanced in time through an explicit TVD Runge-Kutta $3^{\text {rd }}$-order method. It is worth mentioning that unstructured 


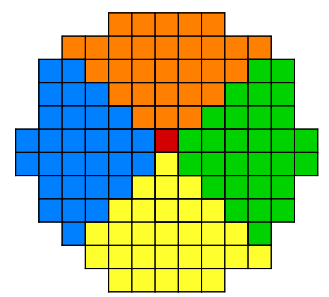

(a) Quadrilateral directional stencils

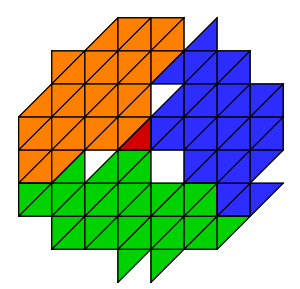

(b) Triangular directional stencils

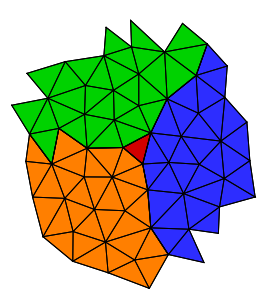

(c) Arbitrary Triangular directional stencils

Figure 3: Unstructured-grid solver multidimensional stencils for a $5^{\text {th }}$ order scheme using different types of elements; considered element in red color.

grids for complex geometries can benefit when combined with variational optimization techniques [34], as well as the use of very high order methods such as the ones proposed in [35].

\subsection{Compressible Lagrange-Remap framework}

The numerical approach of [2] is used in the present study. In particular, a staggered grid arrangement is employed with density $\rho$, internal energy $e$ and pressure $p$ defined at cell centers, and velocity components, $u$ and $v$, defined at cell vertices. The viscous compressible Navier-Stokes equations are solved and the calculation for each time step is divided into two phases. The first phase can be considered as a Lagrangian and the second phase which is the advection one, transports mass, internal energy and momentum across cell boundaries. For low Mach number the Lagrangian phase is divided into several steps, hence the overall time step is thereby less constrained by the sound speed. In the advection phase, the monotonic method of Van Leer $[36,37]$ is used for all fluid variables. It must also be noted that all the flow variables for a given cell at the end of the advection phase lie within the range of values for the cell and its neighbors at the end of the Lagrangian phase. The Lagrange phase is non-dissipative in the absence of shocks. Artificial viscosity, $q$, is used to provide dissipation due to shocks. For the present near-incompressible test problem, this dissipation $(q \nabla u)$ is negligible. As a result the method does not become dissipative at low Mach number. It must be stressed that in order to have the same initial conditions as the other cell-centered frameworks due the staggered grid arrangement the meshes employed are $33 \times 33,65 \times 65$ and $257 \times 257$ points, which are henceforth labelled as $32^{2}, 64^{2}$ and $256^{2}$.

\subsection{Incompressible structured-grid framework}

An incompressible method was also employed in order to compare the low-speed compressible simulations with a reference incompressible solution. The governing equations of conservation of mass and momentum, neglecting the 
effect of the gravity field are written as:

$$
\begin{gathered}
\nabla \cdot \mathbf{u}=0, \\
\frac{\partial \mathbf{u}}{\partial t}+\nabla \cdot(\mathbf{u} \otimes \mathbf{u})=-\frac{1}{\rho} \nabla p+v \nabla^{2} \mathbf{u}
\end{gathered}
$$

where $t$ is the physical time, $\mathbf{u}$ is the velocity field, $p$ is the hydrodynamic pressure, $\rho$ is the fluid density, and $v$ is the kinematic viscosity of the fluid. For the solution of the incompressible equations a method that combines the fractional step (FS) pressure-projection (PP) [38-40] and artificial compressibility method (AC method) [41] has been employed. Details for the unified FSAC-PP method are given by Könözsy and Drikakis [42]. The method has shown superior accuracy and efficiency characteristics compared to the FS-PP and AC approaches for a range of test problems [42, 43]. The characteristics-based (CB) scheme [44-46] is employed for discretizing the convective fluxes. The method falls into the category of pseudo-time splitting FSAC approaches, including a PP step at each pseudo-time step of the dual-time stepping procedure for accelerating the solution towards the incompressibility (divergence-free) constraint. After performing the pseudo-time advancement, the pressure field is computed to update the CB velocity components at each pseudo-time step. By taking the divergence of the semi-discrete equation, similarly to the FS-PP method [39, 47], the cell-centered pressure values are obtained by solving an elliptical pressure-Poisson equation at each pseudo-time step. The numerical solution for the velocity field provides an approximately divergence-free vector field at each pseudo-time step.

The CB scheme has been implemented in conjunction with an upwind $3^{\text {rd }}$ order extrapolation [44-46] (henceforth labeled as U3), as well as with the MUSCL $5^{\text {th }}$ (M5), WENO $5^{\text {th }}$ (W5) and WENO $9^{\text {th }}$ (W9) order schemes. The Gauss-Seidel-type Successive-Over-Relaxation (S.O.R) iteration method [48, 49] is used for solving the discretized elliptical pressure-Poisson equation. The viscous flux terms are approximated by second-order central schemes. The temporal accuracy of pseudo-time stepping procedure can be advanced by applying a Runge-Kutta time integration scheme $[24,45]$.

\section{Double Vortex Statistics}

We first clarify that the double vortex problem is solved in $2 \mathrm{D}$ (defined here as the xy-plane). Thus the $3^{\text {rd }}$ ( $\mathrm{z}$ direction) spatial component is omitted throughout.

Various properties of the double vortex formation and merger are investigated. A passive scalar field $(\varphi)$ is used in order to visualize the formation process of the vortices and subsequent merger. However, the passive scalar can only 
be used for a qualitative comparison between the various schemes and numerical frameworks. In order to conduct quantitative comparisons, the momentum thickness and numerical dissipation are additionally obtained.

The momentum thickness $(\theta)$ is used in order to investigate the growth rate and is calculated as:

$$
\theta=\int_{y=-\infty}^{\infty} \frac{\left[u_{1}-u(y)\right]\left[u(y)-u_{2}\right]}{\left(u_{1}-u_{2}\right)^{2}} d y
$$

where $u_{1}$ and $u_{2}$ are the lower and upper stream velocities. By replacing $u_{1}=\Delta U / 2=-u_{2}$, equation (13) can be re-written as:

$$
\theta=\int_{y=-\infty}^{\infty}\left\{\frac{1}{4}-\left[\frac{u(y)}{\Delta U}\right]^{2}\right\} d y
$$

If the velocity is non-dimensionalized by $\Delta U\left(u_{\infty}\right)$ it is possible to compute the above integral over a domain of size $L \times L$ numerically as:

$$
\theta=\sum_{y=0}^{L}\left[\frac{1}{4}-\bar{u}(y)^{2}\right] \Delta y
$$

where $\Delta y$ is the cell height, and $\bar{u}(y)=\sum_{x=0}^{L}[u(x, y) \Delta x] / L$ is the average velocity along the streamwise direction.

As previously mentioned in the introduction, the numerical methods considered herein are implemented within the framework of ILES. If the flow has not been sufficiently resolved, part of the dissipation of kinetic energy is attributed to the implicit numerical dissipation. In order to assess the performance of the various numerical methods, we quantify and compare the numerical dissipation $\left(D_{N}\right)$ produced by each method. The total loss of kinetic energy at any cell occurs due to the inviscid advection and viscous diffusion. Since viscous diffusion is typically treated by a central order approximation, it does not produce any numerical dissipation. On the contrary, the non-linear advection terms are well known to dissipate kinetic energy when using upwind-type methods. Though there is no known way of quantifying the amount of numerical dissipation at any given cell separately, it is completely plausible to quantify the total kinetic energy loss or dissipation of a closed (isolated) system. The double vortex problem considered here is such a closed system since the boundary conditions do not allow for any kind of transfer, be it mass, momentum or energy, in or out of the domain.

The systems total kinetic energy loss rate $\left(D_{K E}\right)$ due to viscous and numerical dissipation can be evaluated at any time step as follows:

$$
D_{K E}=-\frac{\partial}{\partial t} \int_{y=0}^{L_{y}} \int_{x=0}^{L_{x}} \frac{1}{2} \rho\left(u^{2}+v^{2}\right) d x d y
$$




\begin{tabular}{|c|c|cccc|}
\hline Numerical Framework & Mesh Size & \multicolumn{4}{|c|}{ Reconstruction Scheme } \\
\hline \multirow{2}{*}{$\begin{array}{c}\text { Incompressible } \\
\text { Structured-grid }\end{array}$} & $32^{2}$ & U3 & M5 & W5 & W9 \\
\cline { 2 - 6 } & $64^{2}$ & U3 & M5 & W5 & W9 \\
\cline { 2 - 6 } & $256^{2}$ & - & - & - & W9 \\
\hline \multirow{2}{*}{$\begin{array}{c}\text { Compressible } \\
\text { Structured-grid }\end{array}$} & $32^{2}$ & M3 (+LM) & M5 (+LM) & W5 (+LM) & W9 (+LM) \\
\cline { 2 - 6 } & $64^{2}$ & M3 (+LM) & M5 (+LM) & W5 (+LM) & W9 (+LM) \\
\cline { 2 - 6 } & $256^{2}$ & - & - & - & W9+LM \\
\hline \multirow{2}{*}{$\begin{array}{c}\text { Compressible } \\
\text { Unstructured-grid }\end{array}$} & $32^{2}(\mathrm{QUAD}$ and AT $)$ & M3 & W3 & W5 & W7 \\
\cline { 2 - 6 } & $64^{2}(\mathrm{QUAD}$ and AT $)$ & M3 & W3 & W5 & W7 \\
\cline { 2 - 6 } & $256^{2}$ AT & - & - & - & W7 \\
\hline \multirow{2}{*}{$\begin{array}{c}\text { Compressible } \\
\text { Lagrange-Remap }\end{array}$} & $32^{2}$ & M3 & - & - & - \\
\cline { 2 - 6 } & $64^{2}$ & M3 & - & - & - \\
\cline { 2 - 6 } & $256^{2}$ & M3 & - & - & - \\
\hline
\end{tabular}

Table 1: Numerical schemes used and simulations performed. The schemes are labeled as U (upwind); M (MUSCL); W (WENO); LM (low-Mach); QUAD (Quadrilateral elements); AT (Arbitrary Triangular elements).

The total viscous dissipation $\left(D_{V}\right)$ is given by:

$$
D_{V}=\int_{y=0}^{L_{y}} \int_{x=0}^{L_{x}} \mu\left\{\left(\frac{\partial u}{\partial y}+\frac{\partial v}{\partial x}\right)^{2}+2\left[\left(\frac{\partial u}{\partial x}\right)^{2}+\left(\frac{\partial v}{\partial y}\right)^{2}\right]-\frac{2}{3}\left(\frac{\partial u}{\partial x}+\frac{\partial v}{\partial y}\right)^{2}\right\} d x d y
$$

By having determined the viscous dissipation $\left(D_{V}\right)$ it is now possible to obtain an estimate of the total numerical dissipation:

$$
D_{N}=D_{K E}-D_{V}
$$

It is noted that the above is strictly true only for an incompressible flow. For the weakly compressible case, acoustic modes are present which exchange internal and kinetic energy. Thus this approach of estimating the numerical dissipation $\left(D_{N}\right)$ includes a superimposed oscillation on the true value.

\section{Results}

The computational results of all the numerical frameworks are presented in this section. The schemes are assessed in terms of the vortex evolution pattern (§5.1), momentum thickness (§5.2), numerical dissipation (§5.3) and computational efficiency (\$5.4). Table 1 provides a complete list summarizing all the numerical simulations conducted.

\subsection{Vortex Evolution}

The pattern of the vortex evolution, whereby the growth of unstable waves occurs as a result of the roll up of twodimensional vortical structures interacting with each other to form a single vortical structure, is investigated across all 
the numerical schemes and frameworks encountered in the present study. The main objective is to assess the characteristics of each numerical framework in under-resolved grid arrangements where the impact of the numerical schemes on the vortex formation and structure (flow features resolved) is discussed. To this end, a "reference" simulation obtained by each framework was required in order to ensure that all the methods converge to the same vortex pattern. A very high grid resolution of $256 \times 256$ cells was chosen as the reference resolution for all simulations across the numerical frameworks employed, for which all methods should achieve identical results. As can be seen in Figure 4, where iso-lines of the passive scalar are plotted for values of $0.25,0.5$ and 0.75 , all the schemes provide a similar vortex structure at $t=6.0 \mathrm{~s}$. This is particularly encouraging since it can be regarded as an indication that the flow physics, at this resolution, is not greatly influenced anymore by the numerical schemes employed. Hence, the correct flow pattern (defined as the vortex pattern at the finest resolution) can be presumed to have been captured.

In under-resolved grid arrangements this no longer holds and as a result the different numerical frameworks resolve substantially different vortex structures. As both compressible and incompressible numerical frameworks are employed in the incompressible regime, it is essential to guarantee that the results obtained from the compressible framework are Mach number converged. In other words, it is necessary to ensure that even when performing the simulations at a very low Mach number of 0.02 with a compressible solver, the same vortex pattern is generated as at 0.2 , less some deviations due to small compressibility effects. Moreover, it is critical to investigate the performance of the schemes in this low-Mach number regime. For this purpose an initial qualitative comparison between all the numerical frameworks is undertaken and the performance of each numerical framework is analyzed with regards to the resolved final vortex structure.

In order to assess the effects of low-Mach number dissipation, we have performed incompressible simulations. For the $32 \times 32$ grid (Figure 5), increasing the nominal spatial order of accuracy from $3^{\text {rd }}$ to $9^{\text {th }}$ order only slightly improves the results. In actuality, increasing the nominal accuracy does not result in a higher-order discretization on the coarse grid because the majority of the discretisation stencils, particularly in the case of WENO schemes, encompass the "discontinuity" of the mixing layer, thus the numerical scheme is forced (by design) to reduce its accuracy. As the grid resolution further increases to $64 \times 64$ grid (Figure 6), the effects of the accuracy become more evident, as more detailed flow features inside the merged vortex region become visible.

For the compressible structured-grid framework on the $32 \times 32$ grid (Figure 7), the results are sensitive to the numerical scheme employed. Specifically, the angle of the vortex for the MUSCL $3^{\text {rd }}$ is different than the one obtained by the MUSCL $5^{\text {th }}$, WENO $5^{\text {th }}$ and WENO $9^{\text {th }}$. The compressible structured-grid simulations compare well with the incompressible results on the $32 \times 32$ grid, however, the vortex is more stretched in the WENO $5^{\text {th }}$ and $9^{\text {th }}$ order incompressible simulations. The $64 \times 64$ grid resolution (Figure 8) enables much finer flow features to be resolved 


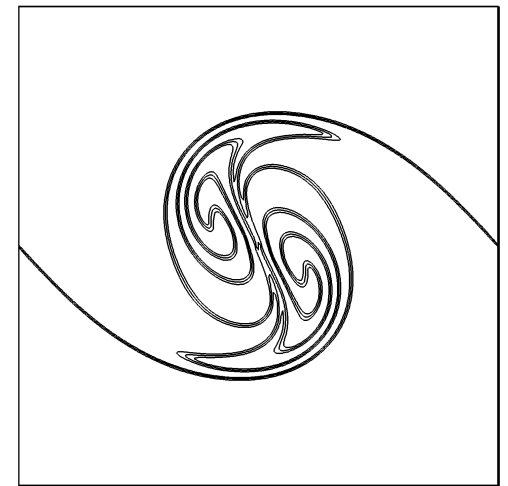

(a) Incompressible Structured-grid (FSACPP) WENO $9^{\text {th }}$ order scheme

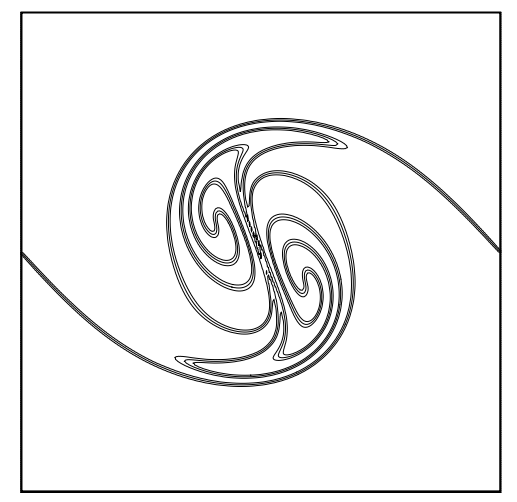

(c) Compressible Unstructured-grid WENO (d) Compressible Lagrange-Remap MUSCL

$7^{\text {th }}$ order scheme (Multi-dimensional stencils, $3^{\text {rd }}$ order scheme Triangular elements)

(b) Compressible Structured-grid WENO $9^{\text {th }}$ order scheme with low-Mach number corerction

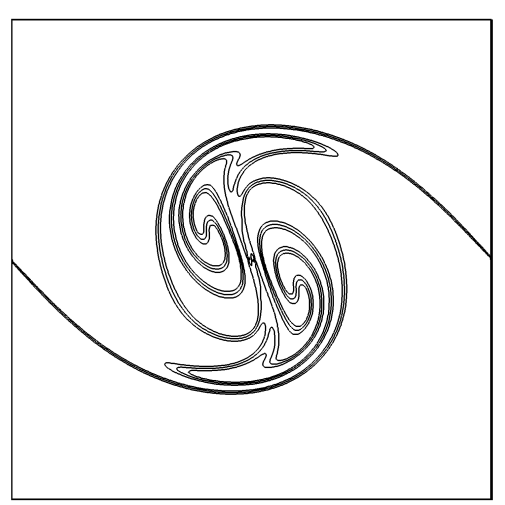

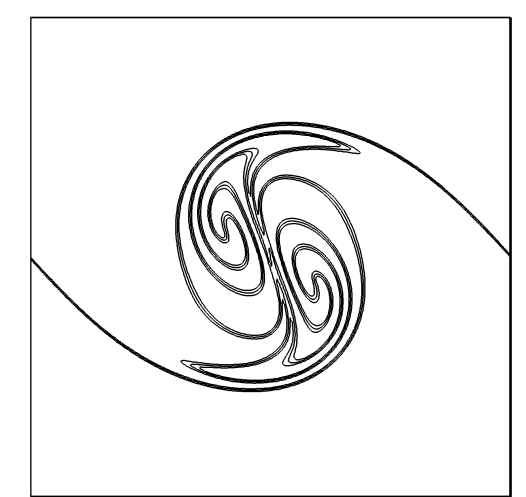

by all examined numerical frameworks for the reference Figure 4: Overview of the final vortex structure obtained by all examined numerical
$256^{2}$ grid resolution at time $t=6.0 \mathrm{~s}$; passive scalar contours $(\mathrm{c}=0.25, \mathrm{c}=0.5, \mathrm{c}=0.75)$.

inside the vortex, which rise in number and detail as the order of accuracy increases (Figure 8(d)).

For the compressible unstructured-grid framework on the $32 \times 32$ grid the results are more sensitive to the numerical scheme employed. For the $32 \times 32$ quadrilateral (QUAD) grid a $3^{\text {rd }}$ order scheme is not capable of resolving the two initial vortices that merge into one single vortex (see Figure 9). On the contrary, the high-order schemes (WENO $7^{\text {th }}$ in particular) are able to capture the correct vortex pattern.

A comparison between quadrilateral (QUAD) and arbitrary triangular (AT) meshes is presented in Figures 10 and 11 for simulations on the $64 \times 64$ grid. The triangular meshes give a greater number of resolved features than those seen by their quadrilateral counterparts. Furthermore, the compressible unstructured-grid framework is more sensitive to the effects of the spatial order of accuracy and the type of grid used than the corresponding structuredgrid framework. The discrepancies between the triangular and quadrilateral meshes can be attributed to the compact 
stencils of the triangular grids[25, 26].

The results for the final resolved vortex structure obtained from the compressible Lagrange-Remap framework are presented in Figure 12. The solution on the $32 \times 32$ grid (Figure 12(a)) has the most accurately formed structure than any of the other numerical frameworks examined, including the incompressible structured-grid simulations, with reference to the highest resolution simulations $\left(256^{2}\right)$. As it will be discussed later in more detail, the implementation of low-Mach corrections in conjunction with the compressible structured-grid solver can lead to similarly accurate results for the vortex structure. On the $64 \times 64$ grid the results obtained by the compressible Lagrange-Remap $3^{\text {rd }}$ order scheme (Figure 12(c)) are very similar to the MUSCL $5^{\text {th }}$ order results of the incompressible and compressible structured-grid simulations of Figures 6(b) and 8(b), respectively.

With regard to the low-Mach number regime, the compressible structured-grid framework is not capable of capturing the correct vortex pattern on the $32 \times 32$ grid using any of the schemes without a low-Mach number correction. This is demonstrated in Figures 13 and 14 for the MUSCL $5^{\text {th }}$ and WENO $9^{\text {th }}$ order respectively. The consequence of reducing the Mach number by an order of magnitude is most apparent for the MUSCL $5^{\text {th }}$ order scheme. At a grid resolution of $64 \times 64$, only the WENO $9^{\text {th }}$ order scheme is capable of capturing the correct vortex pattern; even the MUSCL $5^{\text {th }}$ order scheme does not provide the correct vortex pattern at this resolution unless a low-Mach number correction is used (Figure 13). When the low-Mach number correction is utilized, all schemes are able to provide the correct vortex pattern even on the coarse $32 \times 32$ grid.

The implementation of low-Mach corrections gives a more accurate final vortex structure, specifically on the $32 \times 32$ grid, both for the MUSCL $5^{\text {th }}$ (Figure 13(b)) and WENO $9^{\text {th }}$ order (Figure 14(b)) schemes; compare the above results with the ones in Figures 7(b) and 7(d), respectively. The WENO $9^{\text {th }}$ order scheme is more resilient to the lowMach number dissipation, particularly on the $64 \times 64$ grid (Figure 14(d)). Within the compressible unstructured-grid framework, the WENO $5^{\text {th }}$ order scheme is found to be sufficiently accurate in capturing the correct vortex pattern at low-Mach numbers (Figure 15) without requiring the use of either a low-Mach number correction or preconditioning, even at the lowest grid resolution.

For the compressible Lagrange-Remap framework a $3^{\text {rd }}$ order scheme is capable of resolving the two merged vortices even on the coarse $33 \times 33$ grid (Figure 12). Comparing all numerical frameworks with respect to the vortex evolution patterns over time, the following conclusions can be drawn:

1. At the lowest grid resolution $(32 \times 32)$ the incompressible framework appears insensitive to spatial discretization higher than $5^{\text {th }}$ order. In the compressible structured- and unstructured-grid frameworks a gradual improvement in the results occurs as the order of the numerical schemes is increased.

2. The compressible Lagrange-Remap framework is the only approach that captures the correct vortex pattern with 
a $3^{\text {rd }}$ order numerical scheme on the $33 \times 33$ grid. All the other numerical frameworks require greater resolution, higher-order spatial discretisation, or a combination of both.

3. The only compressible framework that provides the correct vortex pattern in the low Mach regime on a $32 \times 32$ grid resolution without any low-Mach number correction or preconditioning is the unstructured-grid, but only when the WENO $7^{\text {th }}$ order scheme is employed. This can be attributed to the fact that compressible unstructuredgrid is the only numerical framework, where the reconstruction is carried out using characteristic variables and the $k^{\text {th }}$-order WENO schemes of this framework entails the use of the non-linear combination of $(k-1)$-order reconstruction polynomials $([25,26])$ rather than lower-order polynomials as it is the case with the WENO formulation in the structured-grid framework [17].

4. High-order WENO schemes on a $64 \times 64$ grid resolve more flow features than the $3^{\text {rd }}$ order compressible Lagrange-Remap framework; see Figures 6, 8, 10-11 and 12 for the incompressible structured-grid, compressible structured-grid, compressible unstructured-grid and compressible Lagrange-Remap methods, respectively.

5. The compressible structured-grid framework provides sharper and more detailed (better resolved) vortex patterns than the incompressible structured-grid framework for the same scheme and spatial-order of accuracy.

6. The "legs" of the mixing layer are resolved by approximately 1-3 cells irrespective of the grid resolution used. The MUSCL scheme typically requires two to three cells to resolve the "legs" of the mixing layer, while the WENO scheme requires only one to two cells.

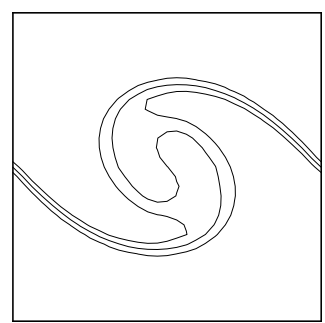

(a) UPWIND $3^{\text {rd }}$

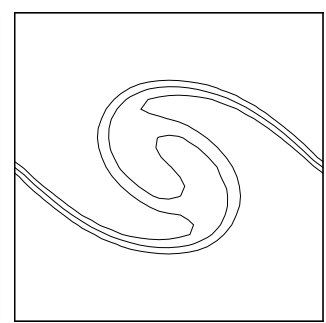

(b) MUSCL $5^{\text {th }}$

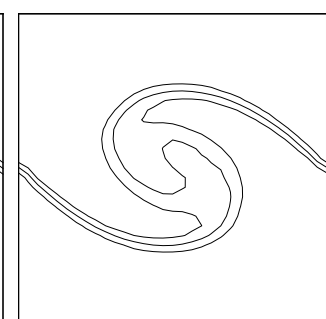

(c) WENO $5^{\text {th }}$

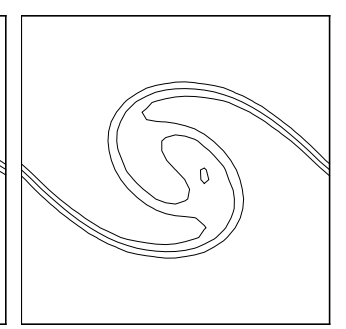

(d) WENO $9^{\text {th }}$

Figure 5: Incompressible structured-grid framework using various limiters on a $32^{2}$ grid resolution at time $t=6.0 \mathrm{~s}$. 


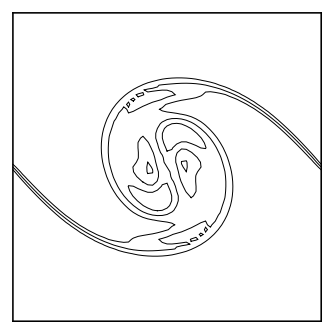

(a) UPWIND $3^{\text {rd }}$

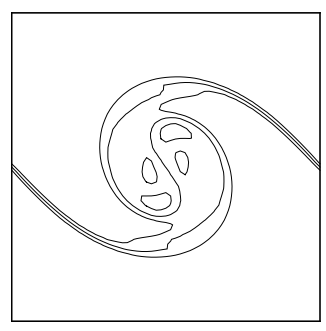

(b) MUSCL $5^{\text {th }}$

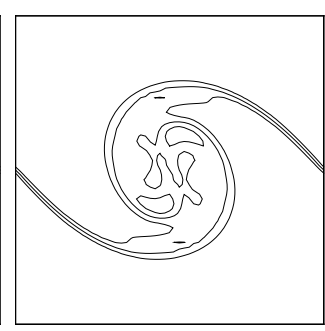

(c) WENO $5^{\text {th }}$

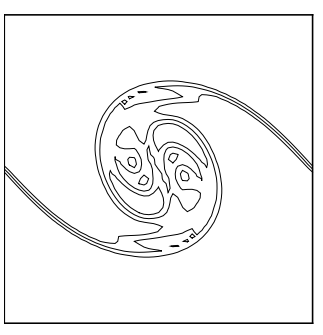

(d) WENO $9^{\text {th }}$

Figure 6: Incompressible structured-grid framework using various limiters on a $64^{2}$ grid resolution at time $t=6.0 \mathrm{~s}$.

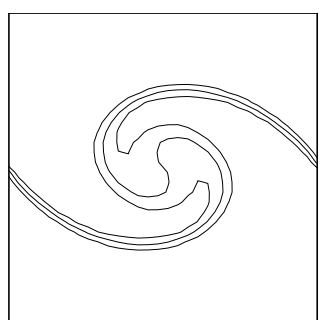

(a) MUSCL $3^{\text {rd }}$

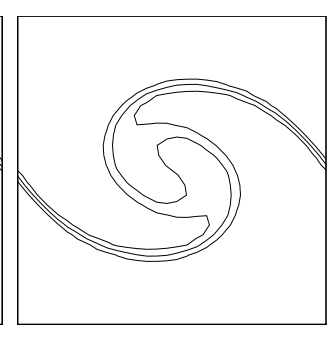

(b) MUSCL $5^{\text {th }}$

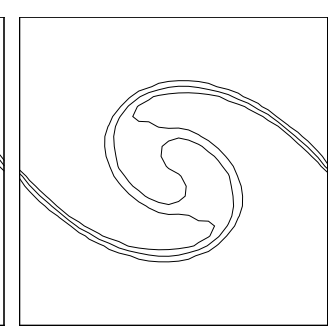

(c) WENO $5^{\text {th }}$

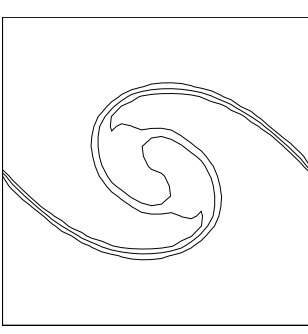

(d) WENO $9^{\text {th }}$

Figure 7: Compressible structured-grid framework using various limiters on a $32^{2}$ grid resolution at time $t=6.0 \mathrm{~s}$.

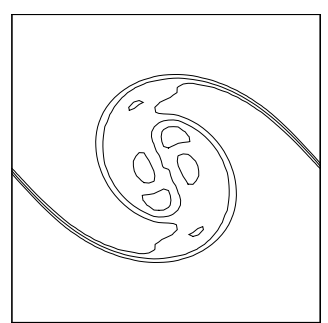

(a) MUSCL $3^{\text {rd }}$

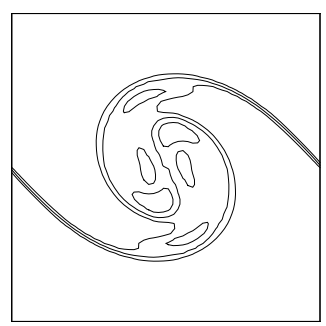

(b) MUSCL $5^{\text {th }}$

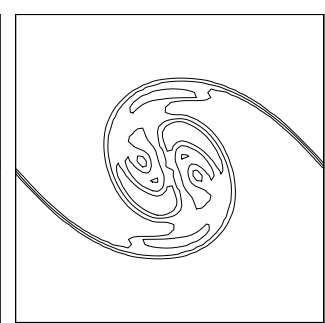

(c) WENO $5^{\text {th }}$

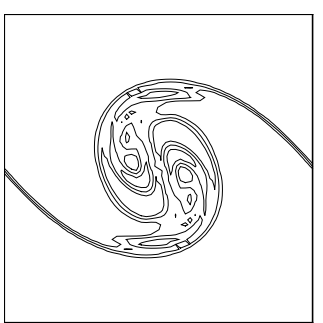

(d) WENO $9^{\text {th }}$

Figure 8: Compressible structured-grid framework using various limiters on a $64^{2}$ grid resolution at time $t=6.0 \mathrm{~s}$. 


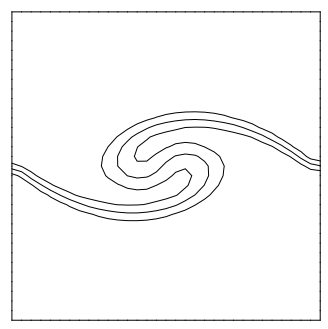

(a) MUSCL $3^{\text {rd }}$

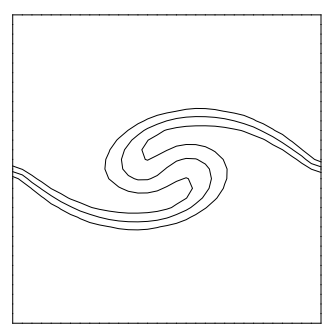

(b) WENO $3^{\text {rd }}$

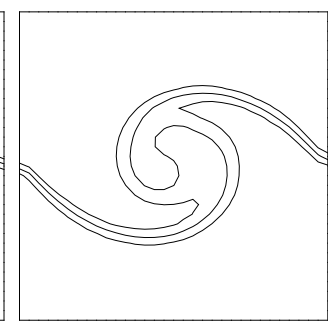

(c) WENO $5^{\text {th }}$

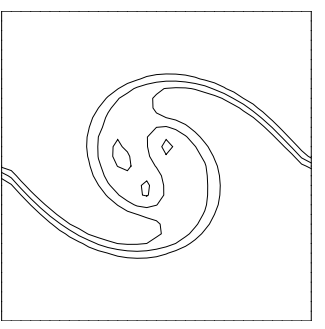

(d) WENO $7^{\text {th }}$

Figure 9: Compressible unstructured-grid framework using various limiters on a $32^{2}$ grid resolution for quadrilateral mesh at time $t=6.0 \mathrm{~s}$.

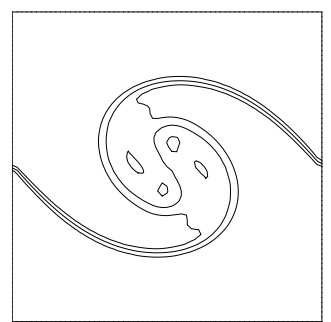

(a) MUSCL $3^{\text {rd }}$

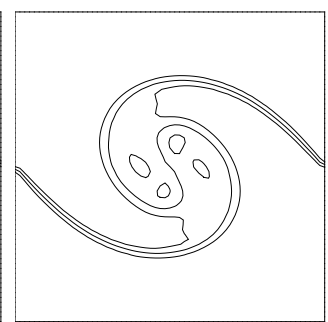

(b) WENO $3^{\text {rd }}$

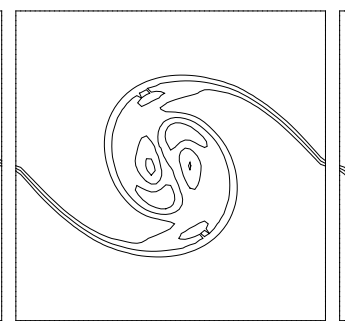

(c) WENO $5^{\text {th }}$

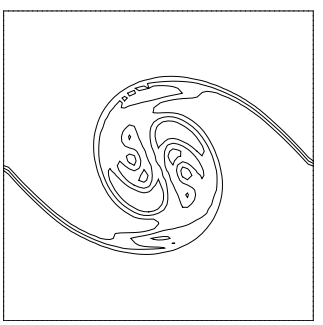

(d) WENO $7^{\text {th }}$

Figure 10: Compressible unstructured-grid framework using various limiters on a $64^{2}$ grid resolution for quadrilateral mesh at time $t=6.0 \mathrm{~s}$.

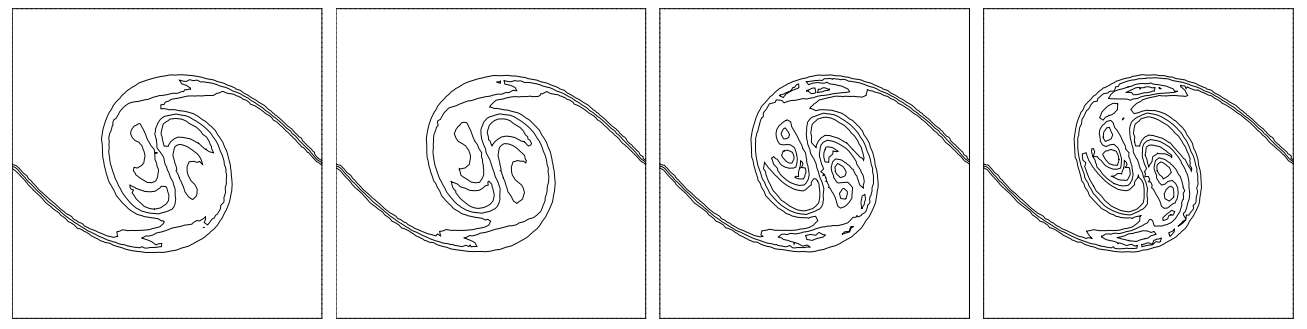
(a) MUSCL $3^{\text {rd }}$
(b) WENO $3^{\text {rd }}$
(c) WENO $5^{\text {th }}$
(d) WENO $7^{\text {th }}$

Figure 11: Compressible unstructured-grid framework using various limiters on a $64^{2}$ grid resolution for Arbitrary Triangular (AT) mesh at time $t=6.0 \mathrm{~s}$. 


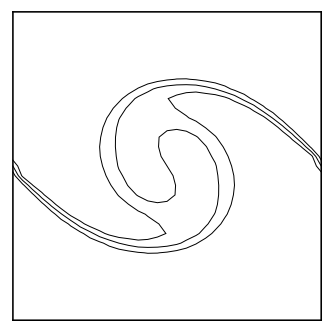

(a) $33 \times 33$

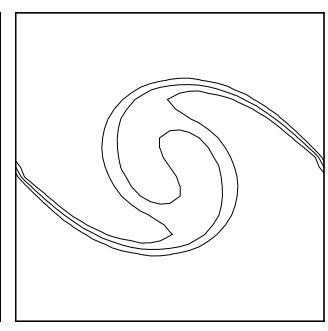

(b) $33 \times 33, \mathrm{Mach}=0.02$

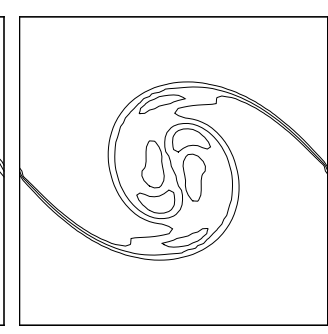

(c) $65 \times 65$

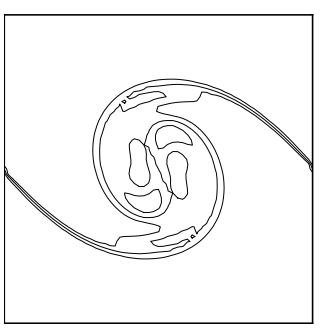

(d) $65 \times 65, \mathrm{Mach}=0.02$

Figure 12: Compressible Lagrange-Remap framework at different grid resolutions and Mach number 0.2 and 0.02 at time $t=6.0 \mathrm{~s}$ and $t=60.0 \mathrm{~s}$ respectively.

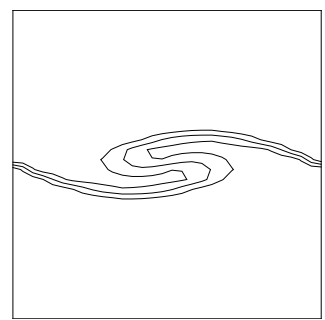

(a) $32 \times 32$

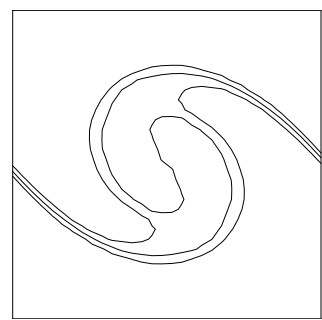

(b) $32 \times 32$, low-Mach number correction

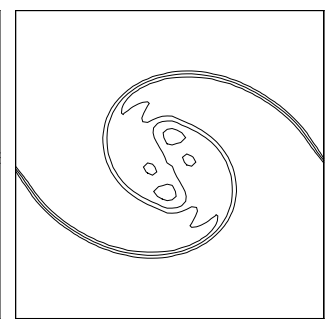

(c) $64 \times 64$

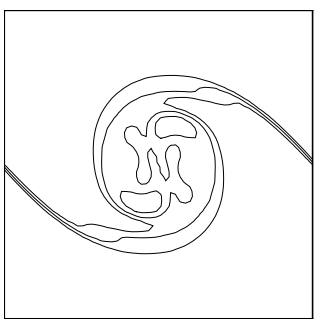

(d) $64 \times 64$, low-Mach number correction

Figure 13: Compressible structured-grid framework at Mach number 0.02 using the MUSCL $5^{\text {th }}$ order scheme at different grid resolutions.

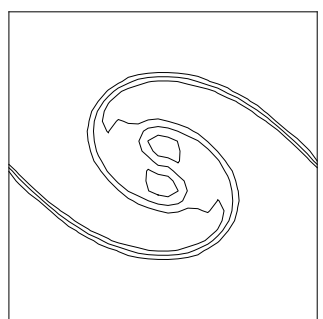

(a) $32 \times 32$

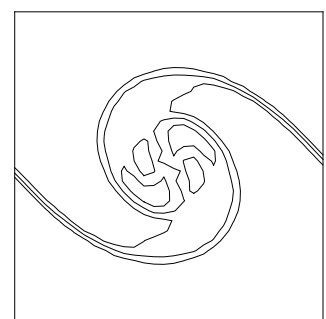

(b) $32 \times 32$, low-Mach number correction

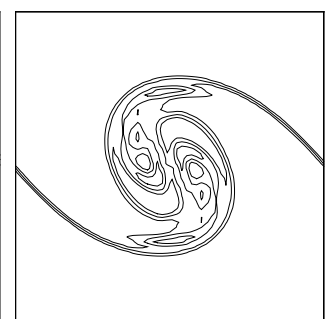

(c) $64 \times 64$

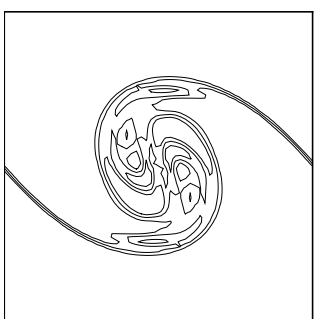

(d) $64 \times 64$, low-Mach number correction

Figure 14: Compressible structured-grid framework at Mach number 0.02 using the WENO $9^{\text {th }}$ order scheme at different grid resolutions. 


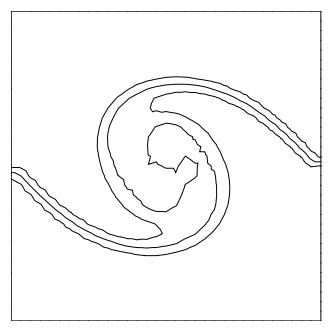

(a) $32 \times 32$, Mach $=0.2$

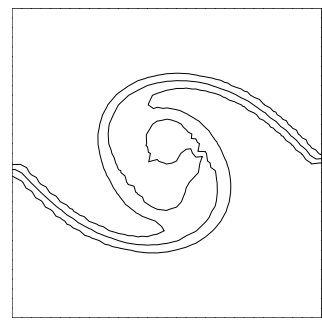

(b) $32 \times 32, \mathrm{Mach}=0.02$

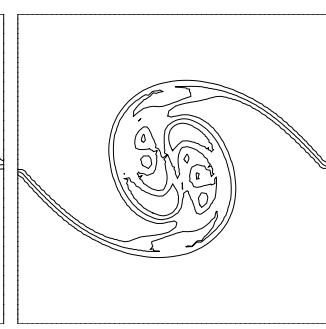

(c) $64 \times 64, \mathrm{Mach}=0.2$

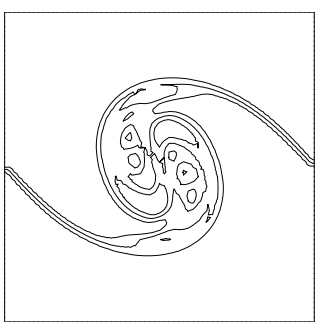

(d) $64 \times 64, \mathrm{Mach}=0.02$

Figure 15: Compressible unstructured-grid framework using the WENO $5^{\text {th }}$ order scheme at different Mach number and grid resolutions (Arbitrary Triangular elements).

\subsection{Momentum Thickness}

The double vortex pairing problem under examination provides an ideal test case for evaluating the performance of the various numerical schemes and frameworks since the resolved momentum thickness growth from the initial shear layer is a distinctive characteristic of the mixing in the shear layer. Moreover, a quantitative assessment of the accuracy of all the schemes can be performed using this metric. As in the previous section commenting on vortex evolution, all of the numerical frameworks employed are compared against a reference simulation conducted at a grid resolution of $256 \times 256$ in order to ensure that the methods converge to the same momentum thickness growth patterns.

The FSAC-PP method was used to obtain all the results (Figure 16) in the incompressible framework. Increasing the spatial-order of accuracy provided closer agreement with the momentum thickness patterns of the reference simulations. One of the most important observations is that the momentum thickness at the end of the simulation $t=6.0 \mathrm{~s}$ is not monotone converging for the $32^{2}$ grid as shown in Figure 16(a) while it must also be stressed that at the same grid resolution, none of the schemes within the incompressible framework agree with the non-linear mixing layer evolution regime from time $t=1.0 \mathrm{~s}$ to $t=3.0 \mathrm{~s}$ of the reference simulation. In contrast, the higher resolution $64 \times 64$ grid shows a much better agreement to the reference simulation for all methods, suggesting that at the lower resolution of $32^{2}$, the effect of the numerical dissipation in conjunction with the initial unresolved interface discontinuity is more profound.

With regards to the compressible structured-grid framework, at a $64 \times 64$ grid resolution not all schemes are capable of providing a momentum thickness evolution pattern that is in close agreement to the reference simulation. In particular, without the low-Mach number correction the momentum thickness is over predicted at late times for the MUSCL $5^{\text {th }}$ order scheme as seen in Figure 17(a). The implementation of the low-Mach number correction has resulted in an identical temporal growth rate of the momentum thickness at Mach numbers 0.2 and 0.02 . Without the low-Mach number correction the momentum thickness at late times deviates severely from the reference simulation, apart of the WENO $9^{\text {th }}$ order scheme on a $64 \times 64$ grid resolution (Figure 17(b)). 
For the compressible unstructured-grid framework, the results obtained (see Figure 18) indicate that it is also sensitive to the numerical scheme and type of grid employed. For the $32 \times 32$ quadrilateral grid, the $3^{\text {rd }}$ order scheme is not capable of resolving the two initial vortices, hence it also under-predicts the momentum thickness at late times. However, the higher-order schemes such as the WENO $5^{\text {th }}$, provide improved accuracy and the results are therefore in closer agreement with the reference simulation. This is due to the fact that at the higher-spatial order of accuracy both vortices become resolved. On a $32 \times 32$ grid using a triangular mesh the results improve dramatically and a good agreement with the reference simulation is achieved. Furthermore, the non-linear mixing layer evolution between $t=1.0 \mathrm{~s}$ and $t=3.0 \mathrm{~s}$ is well captured by the WENO $5^{\text {th }}$ order scheme for both grid resolutions employed.

At the $64 \times 64$ quadrilateral grid resolution, the only scheme capable of capturing the non-linear mixing layer evolution is the WENO $5^{\text {th }}$ order. The triangular meshes can capture this regime with all numerical schemes employed here apart from the WENO $3^{\text {rd }}$ order at the the $32 \times 32$ mesh. The compressible unstructured-grid framework is sensitive to the spatial-order of accuracy in conjunction with the type of mesh used. Quadrilateral meshes require higher-order of spatial discretization in order to produce similar results to triangular meshes. This behavior is due to the non-local character of the reconstruction associated with the quadrilateral stencils that extend to a greater region on a given mesh than the corresponding triangular ones. Using the WENO $5^{\text {th }}$ order scheme on quadrilateral element meshes provides the most accurate results. It appears that the most influential parameter that drives the performance of multi-dimensional reconstruction is not the compactness of the stencils, but the orientation of the cells with respect to flow features. Consequently, the arbitrary nature of the unstructured triangular meshes proves beneficial for resolving complicated flow features that are usually arbitrary in terms of orientation and structure. The full mechanism for this is as yet not fully understood. Another significant feature of the compressible unstructured-grid framework is that the WENO $5^{\text {th }}$ order scheme provides sufficient accuracy to obtain the same momentum thickness evolution pattern as the reference simulation in the low-Mach number regime (Figure 18) even without resorting to low-Mach correction or preconditioning.

For the compressible Lagrange-Remap framework (Figure 19) the momentum thickness calculated on the $33 \times 33$ grid is under-predicted. Using a $65 \times 65$ grid resolution, a closer agreement with the reference simulation is achieved (Figure 19). Although the momentum thickness on the $65 \times 65$ grid resolution is quite similar to the reference simulation, the non-linear mixing layer evolution regime from time $t=1.0 \mathrm{~s}$ to $t=3.0 \mathrm{~s}$ is under-predicted. The same trend also occurs during the very low-Mach number simulation, where good agreement with the reference simulation is achieved (hardly distinguishable from the reference simulation at Mach number of 0.2).

The main conclusions regarding the accuracy of different numerical frameworks with respect to the momentum thickness growth are summarised below: 
1. The prediction of momentum thickness is sensitive to the spatial-order of accuracy with higher accuracy achieved when increasing the order of accuracy.

2. The compressible unstructured-grid framework is capable of capturing the momentum thickness evolution at low-Mach number even without the use of a low-Mach number correction or preconditioning.

3. The compressible structured-grid framework is prone to low-Mach dissipation, except the case of WENO $9^{\text {th }}$ order scheme on the $64 \times 64$ grid.

4. All the schemes, in all the frameworks provide non-monotone converging results for momentum thickness towards the end of the simulation. This indicates a dependence upon the initial conditions varying with the grid resolution;

5. The most difficult pattern to accurately capture at the coarsest resolution $(32 \times 32)$ is the non-linear mixing layer regime between $t=1.0 \mathrm{~s}$ and $t=3.0 \mathrm{~s}$. This is indicative of the secondary eigenmode of the initial condition associated with the second wavenumber $k_{2}$ and the formation of the two pairing vortices.

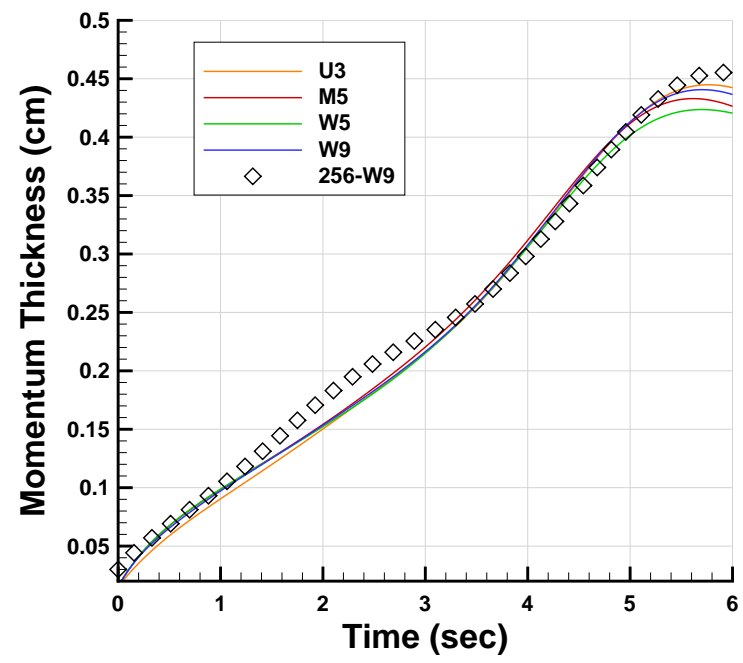

(a) $32 \times 32$

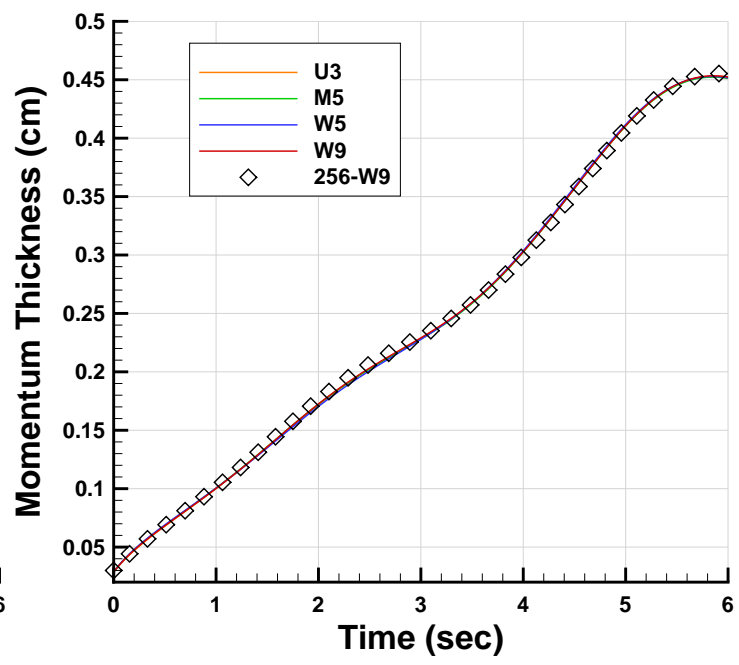

(b) $64 \times 64$

Figure 16: Momentum thickness in time for the incompressible structured-grid framework using various numerical schemes and grid resolutions. 


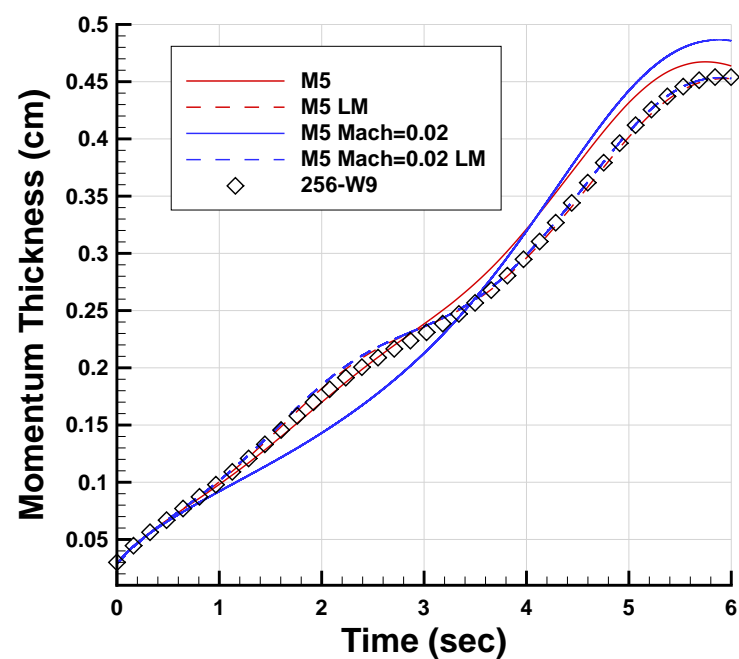

(a) MUSCL-5 $5^{\text {th }}$

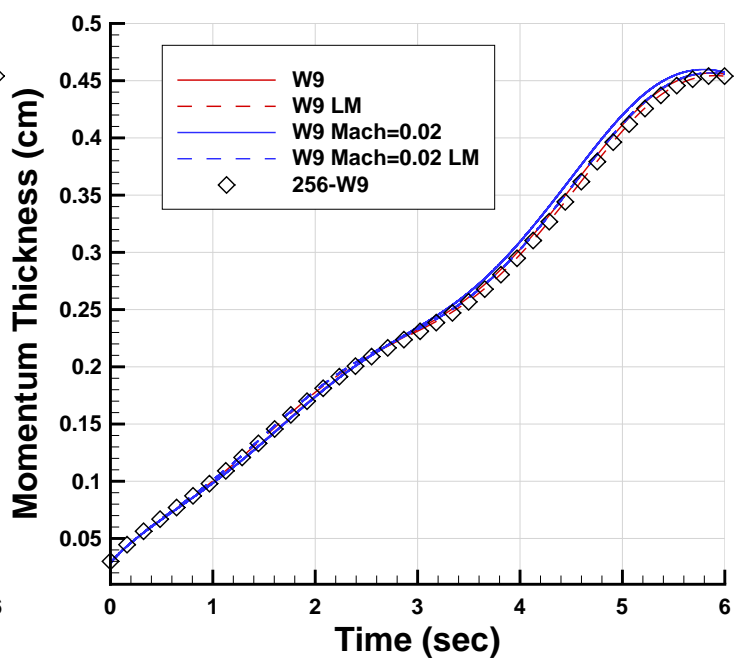

(b) WENO-9 ${ }^{\text {th }}$

Figure 17: Momentum thickness in time for the compressible structured-grid framework using various numerical schemes and Mach number on the $64^{2}$ grid.

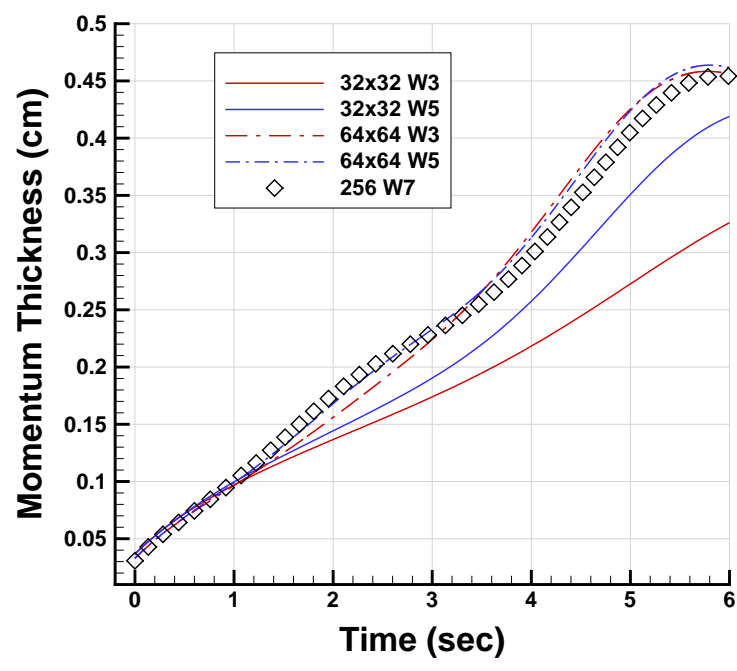

(a) Quadrilateral

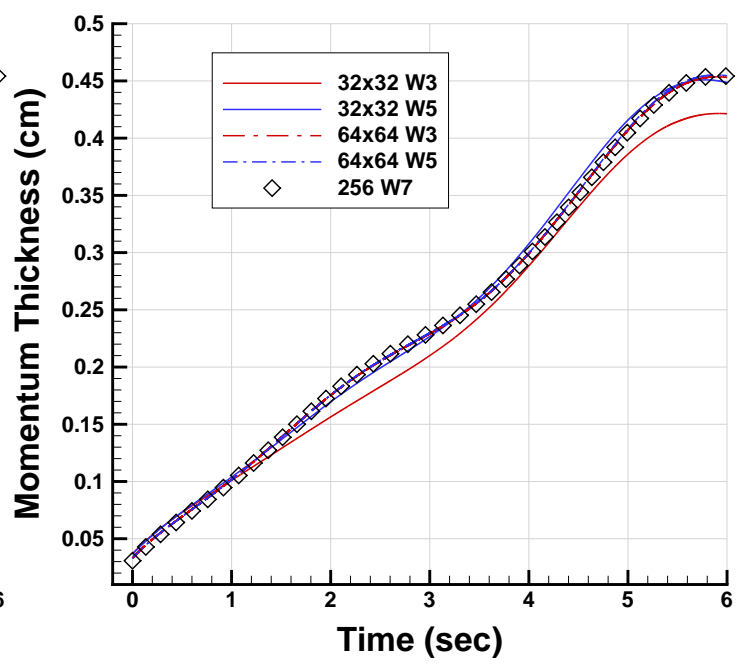

(b) Triangular

Figure 18: Momentum thickness variation in time for the compressible unstructured-grid framework using various numerical schemes, grid resolutions and element types. 


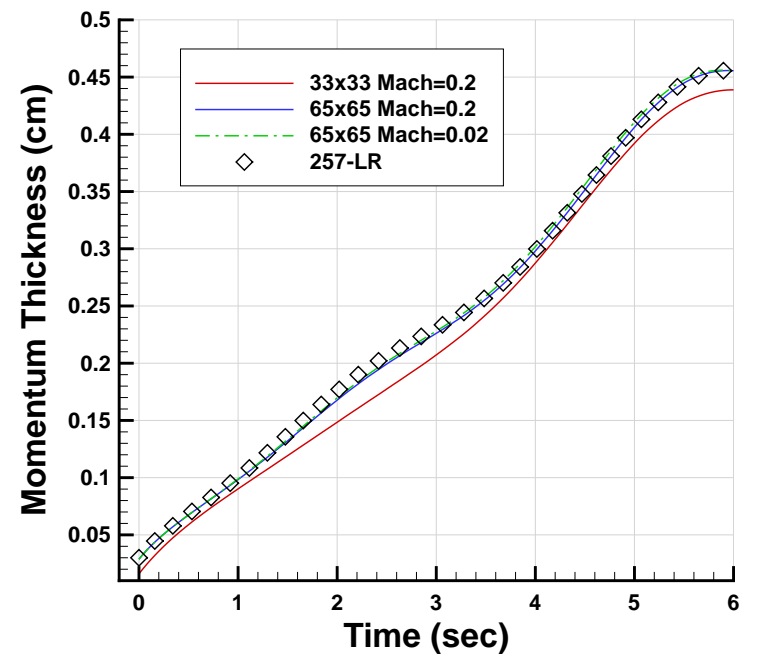

Figure 19: Momentum thickness variation in time for the compressible Lagrange-Remap framework using various grid resolutions and Mach numbers.

\subsection{Numerical Dissipation}

The viscous dissipation $D_{V}$ (equation (17)) and kinetic energy loss rate $D_{K E}$ (equation (16)), or total dissipation, provide the means to investigate the numerical dissipation $D_{N}$ (equation (18)) of each computational framework at the various grid resolutions. In the incompressible case, $D_{K E}$ represents the total dissipation rate (viscous plus numerical). In the compressible cases, $D_{K E}$ represents the total dissipation rate on average, but there is superimposed oscillatory behavior due to acoustic vibrations, as will be discussed later. An example showcasing the contribution of each dissipation component is given in Figure 20. The dissipation density (per volume) in Figures 20-26 is measured in $\mathrm{kg} /\left(\mathrm{m} \cdot \mathrm{s}^{3}\right)$.

In the incompressible simulations at the $32 \times 32$ resolution, the numerical dissipation of the $3^{\text {rd }}$ order upwind scheme initially has lower values, while the MUSCL $5^{\text {th }}$, WENO $5^{\text {th }}$ and $9^{\text {th }}$ order schemes have a higher numerical dissipation that reduces, however, at a faster rate (Figure 21(a)). At $64 \times 64$ (Figure 21(b)) resolution the dissipation changes similarly for all schemes, leading to the conclusion that the numerical reconstruction has a little influence on the incompressible FSAC-PP method.

For the compressible structured-grid framework at $32 \times 32$ resolution (Figure 22) it is evident by comparing the MUSCL $5^{\text {th }}$ (Figure 22(a)) and WENO $9^{\text {th }}$ (Figure 22(b)) order schemes that the numerical dissipation is reduced as the spatial order of accuracy is increased, thus obtaining a closer agreement with the reference simulation.

The oscillatory behavior of the numerical dissipation at Mach number of 0.2 is due to acoustic vibrations. Reducing the Mach number value to 0.02 significantly reduces the oscillations. Employing the low-Mach number correction at Mach number of 0.02 is found to significantly reduce the numerical dissipation $\left(D_{N}\right)$ on $32^{2}$ (Figure 22) and $64^{2}$ 


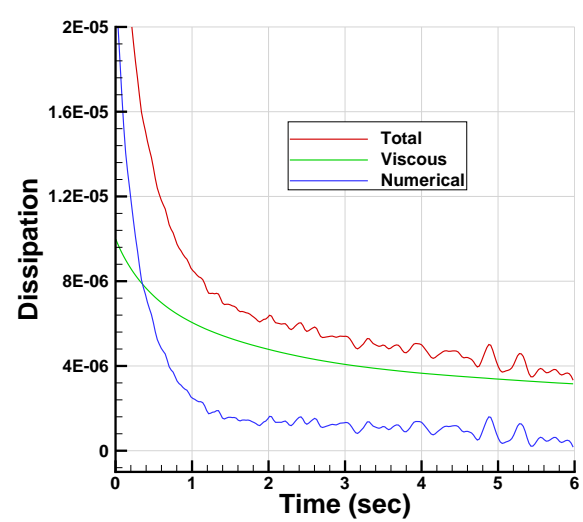

(a) $32^{2}$ MUSCL $5^{\text {th }}$

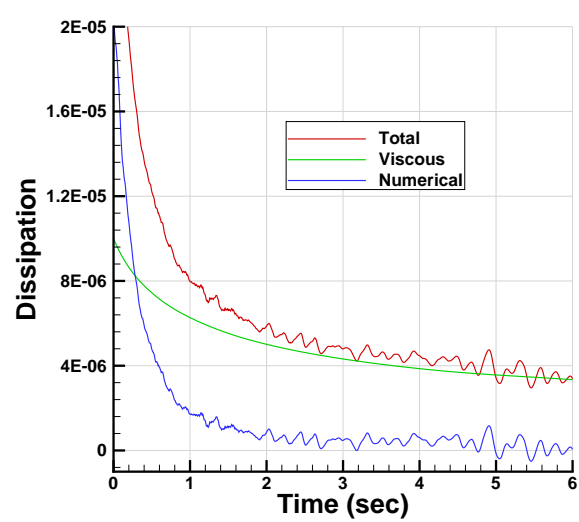

(c) $32^{2}$ WENO $9^{\text {th }}$

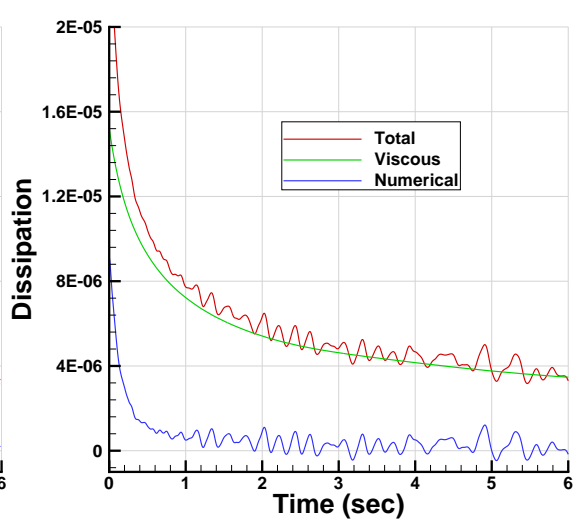

(b) $64^{2}$ MUSCL $5^{\text {th }}$

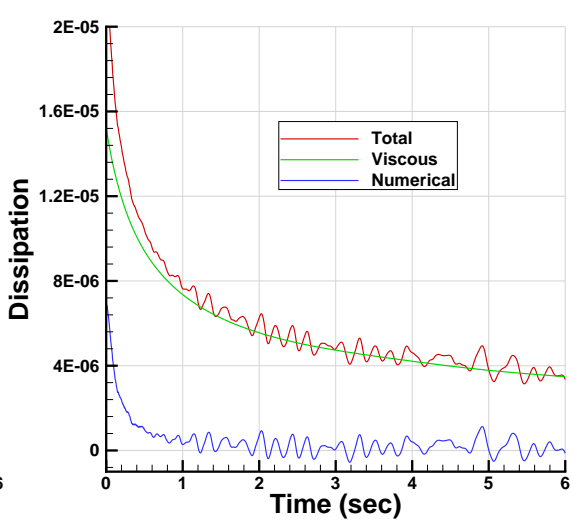

(d) $64^{2}$ WENO $9^{\text {th }}$

Figure 20: Comparison of total $D_{K E}$, viscous $D_{V}$ and numerical $D_{N}$ dissipation for the MUSCL $5^{\text {th }}$ and WENO $9^{\text {th }}$ order schemes on the $32^{2}$ and $64^{2}$ grids in the compressible structured-grid framework.

(Figure 23) grid resolutions for almost all schemes. Specifically, all schemes are found to have a better match to the reference solution when utilizing the low-Mach number correction, except the WENO $9^{\text {th }}$ order scheme for which no tangible changes appear.

Using the compressible structured-grid finite volume solver to perform ILES of a turbulent plane channel flow [50], it was observed that the low-Mach number correction in conjunction with the WENO $9^{\text {th }}$ order scheme had an adverse effect on the accuracy of the solution, similar to what is observed herein. However, the results on the very coarse grid $(32 \times 32)$ actually show an improvement when using the low-Mach number correction with the WENO $9^{\text {th }}$ order scheme suggesting that there is a more complex interaction of the numerical terms. This is demonstrated qualitatively by the improvement in the structure and resolved features of the final vortex compared to the cases; compare the results with and without low-Mach correction in (Figure 14(b)) and (Figure 14(a)), respectively, as well as the reduction in the numerical dissipation in (Figure 22(b)). The results of Figure 22(b) show that the numerical 
dissipation is significantly reduced during the first second of the double vortex instability development. Hence, in under-resolved simulations encompassing significant numerical dissipation the use of the low-Mach correction is likely to prove overall beneficial.

Figure 23 presents the numerical dissipation on the $64 \times 64$ grid for the compressible structured-grid framework. The comparison of the results in Figures 23(a) and 23(b) show that for Mach number of 0.2 the MUSCL $5^{\text {th }}$ order scheme is only slightly more dissipative than the WENO $9^{\text {th }}$ order. However, the difference between the two schemes becomes more apparent when reducing the Mach number to 0.02. The WENO $9^{\text {th }}$ order shows hardly no difference to the (average) dissipation values obtained at Mach number of 0.2. The implementation of the low-Mach number correction at a Mach number of 0.2 manages to marginally reduce the numerical dissipation of the MUSCL $5^{\text {th }}$ order scheme (Figure 23(a)). At a Mach number of 0.02, however, the reduction of numerical dissipation is even more profound. The difference in the results of the WENO $9^{\text {th }}$ order scheme with and without low Mach correction are practically indistinguishable (Figure 23(b)).

For the compressible unstructured-grid framework at $32 \times 32$ resolution (Figure 24) the numerical dissipation relies on the spatial-order of accuracy of the scheme throughout the simulation. As the spatial-order of accuracy is increased, a closer agreement with the reference simulation is obtained. All the numerical reconstructions exhibit the oscillatory dissipation behavior. At the $64 \times 64$ grid resolution, the numerical dissipation $\left(D_{N}\right)$ approaches zero much faster at early times comparing to the remaining computational approaches, while simultaneously the oscillatory behavior becomes less pronounced, especially when using triangular shaped elements (Figure 25).

For a structured (quadrilateral) mesh, the numerical dissipation of the compressible structured-grid framework using the WENO $9^{\text {th }}$ order scheme and that of the compressible unstructured-grid framework using the WENO $5^{\text {th }}$ order scheme (multi-dimensional stencil) are very similar (Figure 25). However, for (arbitrary) triangular elements the compressible unstructured-grid framework exhibits significantly lower numerical dissipation, particularly during the first few seconds of the vortex evolution, where the two pairing vortices develop. To some extent this explains why the compressible unstructured-grid framework is capable of obtaining the most accurate final vortex structure at the very low-Mach number of 0.02 discussed in $§ 5.1$ and Figure 15; the compressible-unstructured framework is able to adequately resolve the secondary instability (second eigenmode in equation (3)), which develops into the two pairing vortices, as a result of the very low numerical dissipation.

Similar to other methods, in the compressible Lagrange-Remap framework the numerical dissipation $\left(D_{N}\right)$ reduces with increasing the mesh size (Figure 26). However, the values of the numerical dissipation $\left(D_{N}\right)$ attained at early times are significantly lower than any of the other compressible frameworks. The oscillations witnessed in the the total dissipation at the Mach number of 0.2 are attributed to acoustic effects; again the oscillations are significantly 
reduced at the Mach number of 0.02 .

Comparing all the numerical frameworks with respect to the numerical dissipation lead to the following conclusions:

1. The oscillatory behavior of the numerical dissipation $\left(D_{N}\right)$ is a consequence of the oscillations present in the total dissipation $\left(D_{K E}\right)$ as shown in Figure 20. These oscillations occur only in the compressible solutions due to acoustic (compressible) effects as demonstrated in Figure 26(b). At $256^{2}$ resolution, the numerical dissipation is almost purely comprised of the "resolved" acoustic fluctuation, as it is evident by the very close agreement between the different compressible frameworks, particularly after $t=1 \mathrm{sec}$. An almost perfect agreement is obtained between the compressible structured- and unstructured-grid frameworks. In the compressible Lagrange-Remap framework a good agreement is present at early times, however, a phase difference is evident at late times.

2. At $32 \times 32$ grid resolution, the compressible unstructured-grid framework provides the best agreement with the reference simulation. As the spatial order of accuracy is increased a better agreement is achieved even at earlier times. This can be attributed to the fact that the present framework utilizes the $(k-1)$ order polynomials for a $k^{\text {th }}$ order accurate schemes, and that the same polynomials are used for the approximation of the velocity and temperature gradients in the viscous fluxes. For comparison, both the incompressible and compressible structured-grid frameworks use a $2^{\text {nd }}$ order central approximation of the temperature and velocity derivatives in the viscous fluxes and are thus second order accurate. Clearly, there is a benefit of employing higher-order approximations for the viscous part of the equations.

3. The compressible Lagrange-Remap framework provides a closer agreement with the reference simulation at early times than the compressible structured-grid framework. 


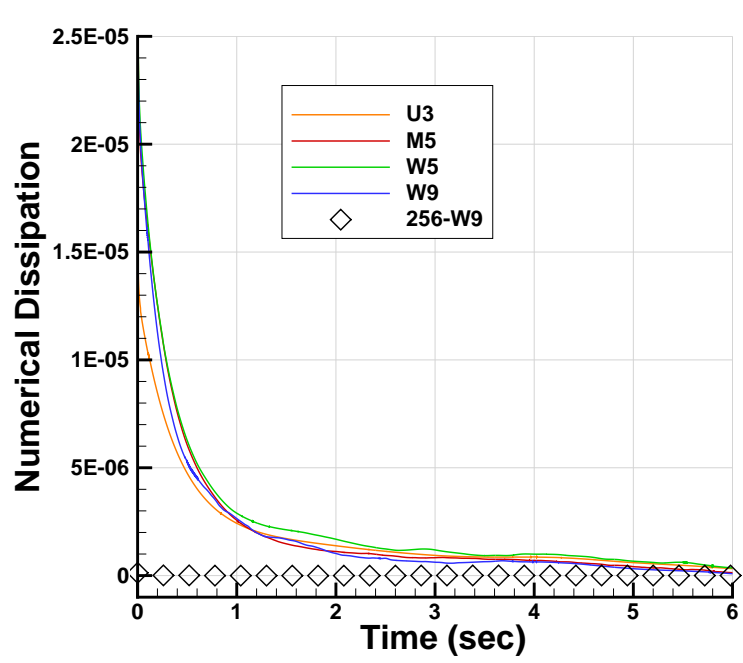

(a) $32 \times 32$

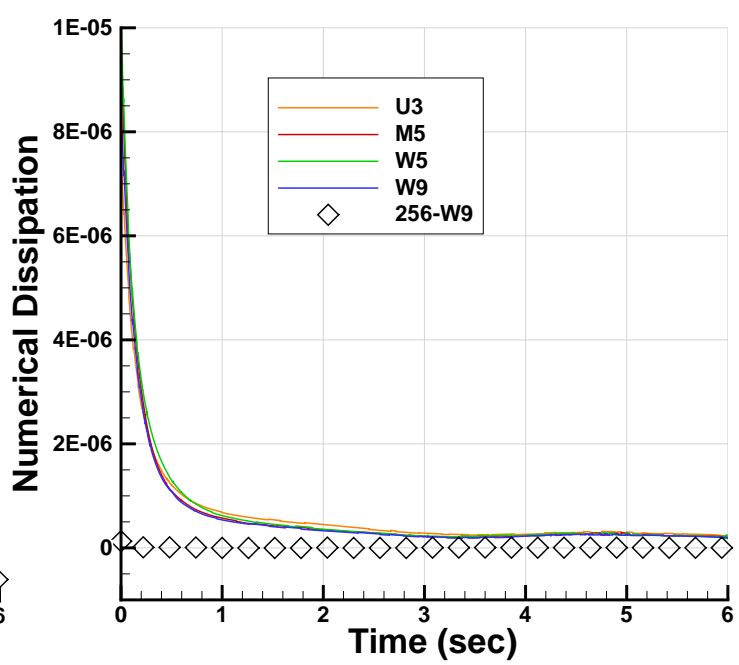

(b) $64 \times 64$

Figure 21: Numerical dissipation in time for the incompressible structured-grid framework using various numerical schemes and grid resolutions.

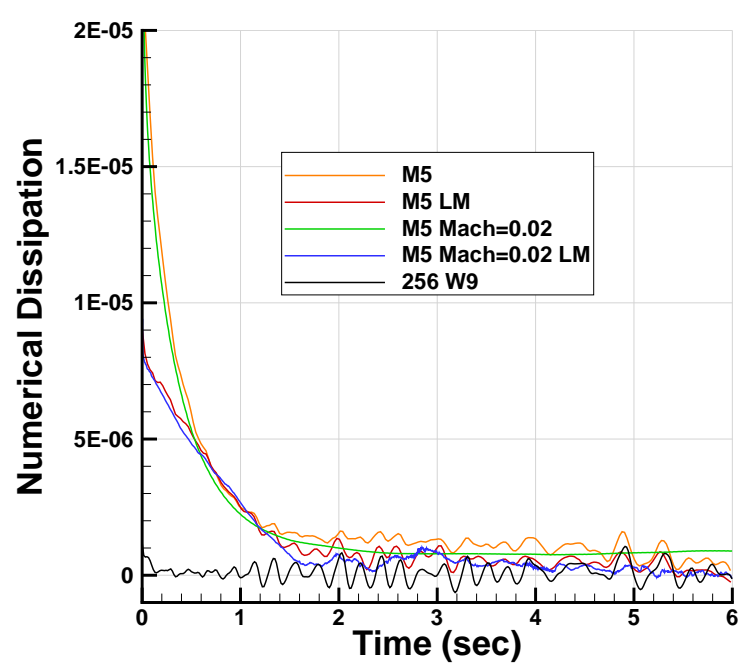

(a) MUSCL $5^{\text {th }}$

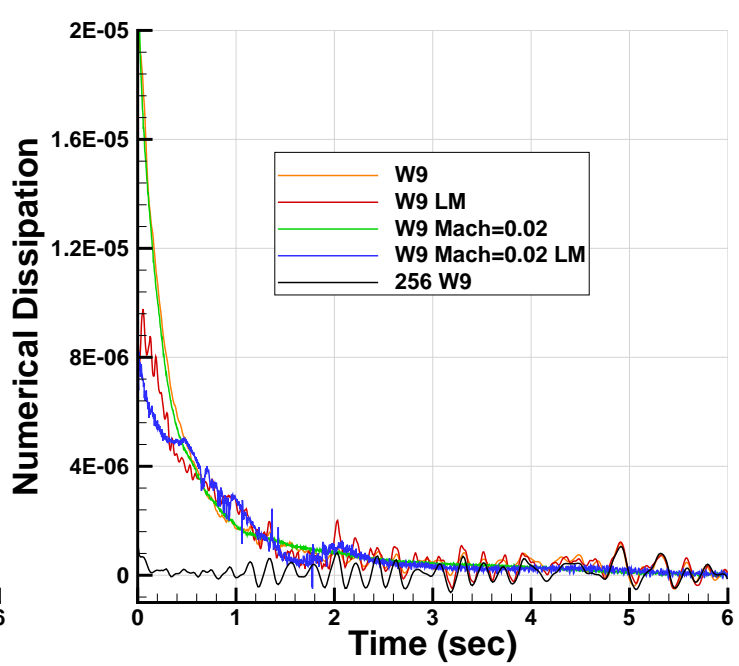

(b) WENO $9^{\text {th }}$

Figure 22: Numerical dissipation in time for the compressible structured-grid framework using various numerical schemes on the $32 \times 32$ grid. 


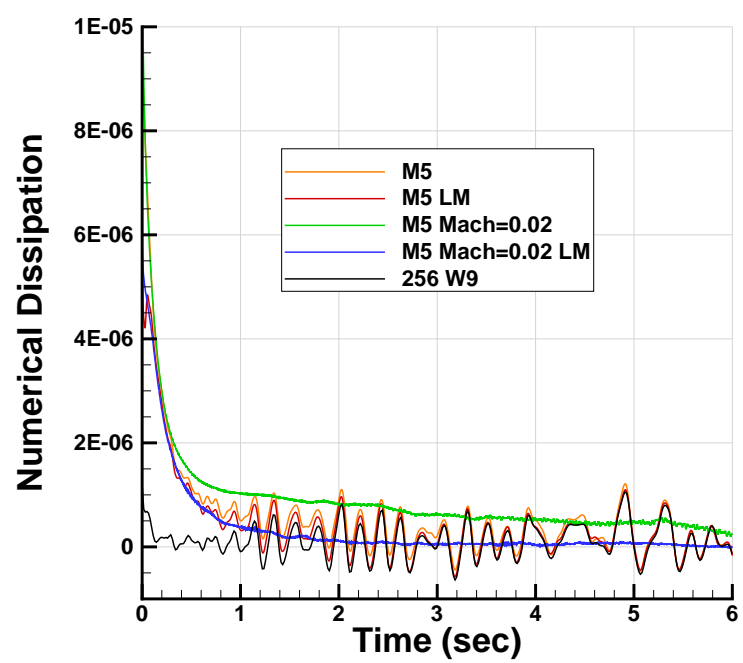

(a) MUSCL $5^{\text {th }}$

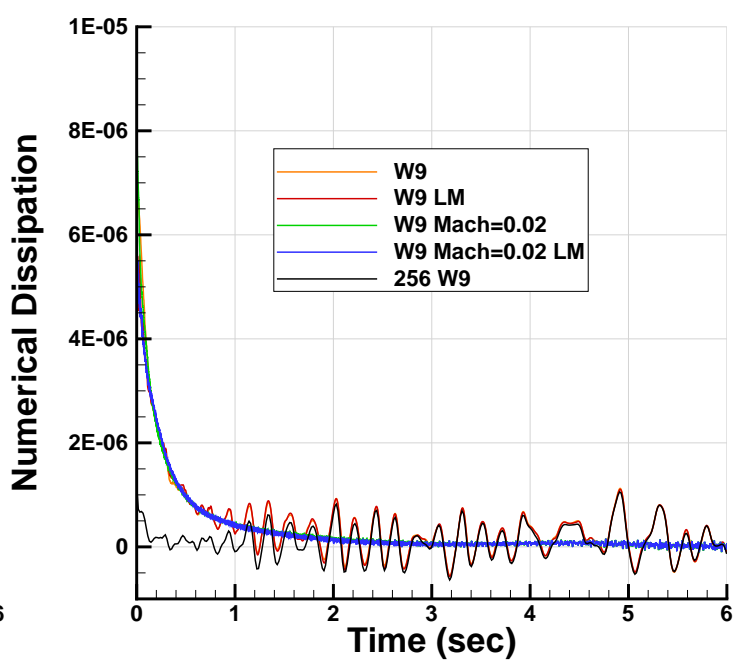

(b) WENO $9^{\text {th }}$

Figure 23: Numerical dissipation in time for the compressible structured-grid framework using various numerical schemes on the $64 \times 64$ grid.

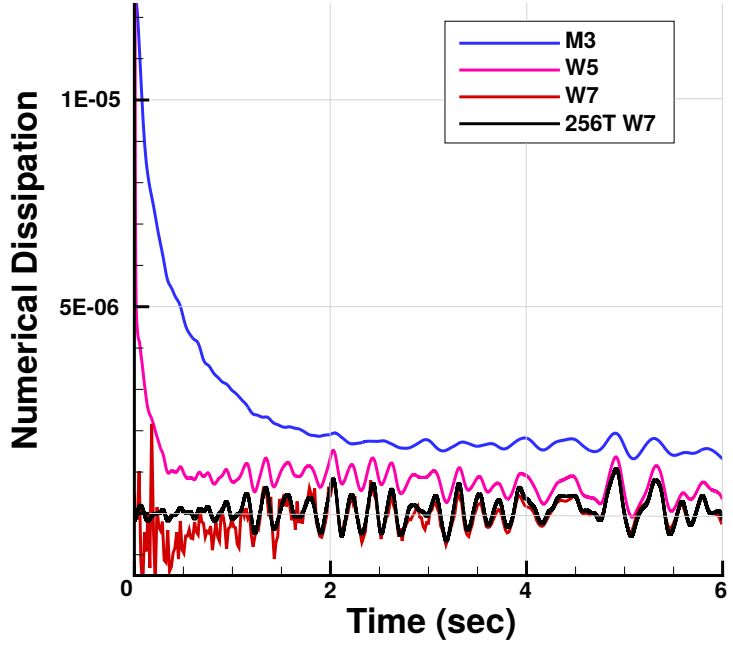

(a) $32 \times 32$

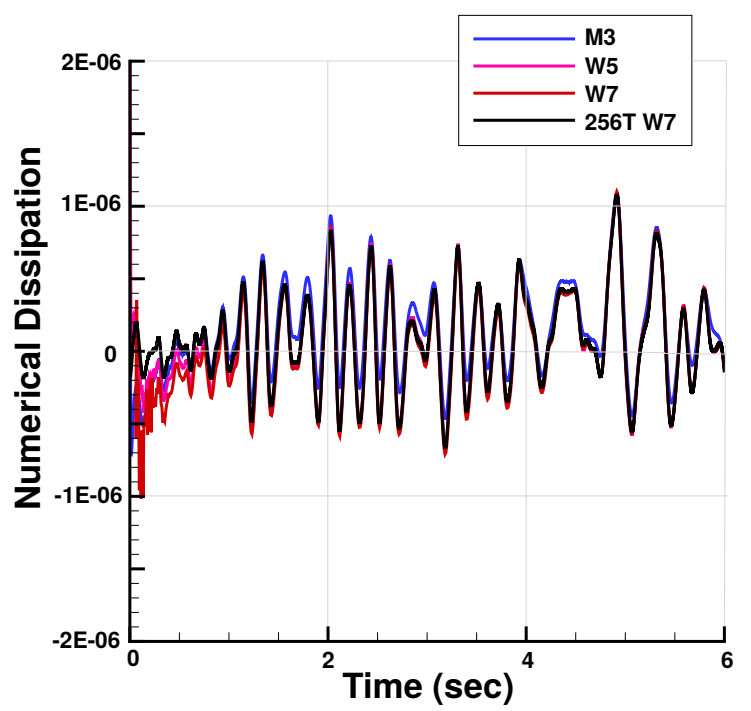

(b) $64 \times 64$

Figure 24: Numerical dissipation for the compressible unstructured-grid framework (Triangular elements). 


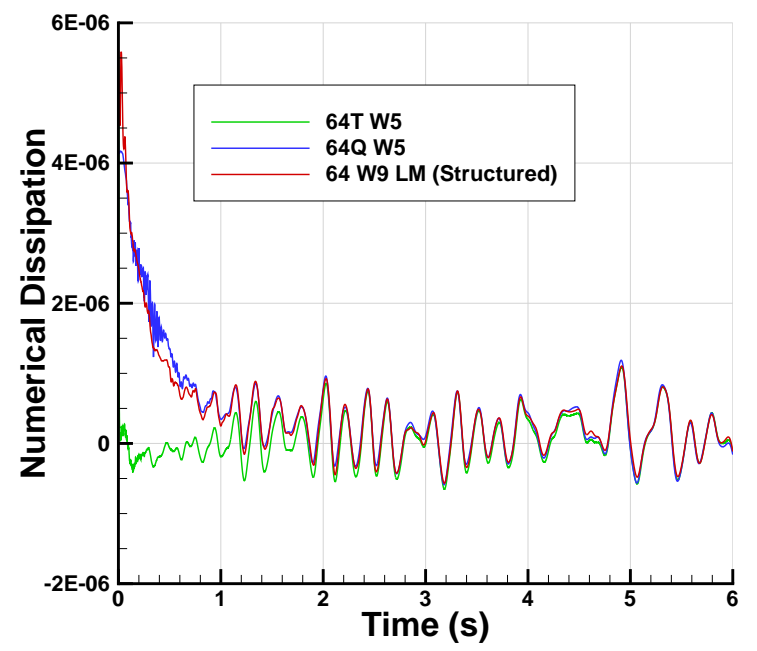

Figure 25: Numerical dissipation for the compressible unstructured-grid framework; comparison between Quadrilateral vs. Triangular elements.

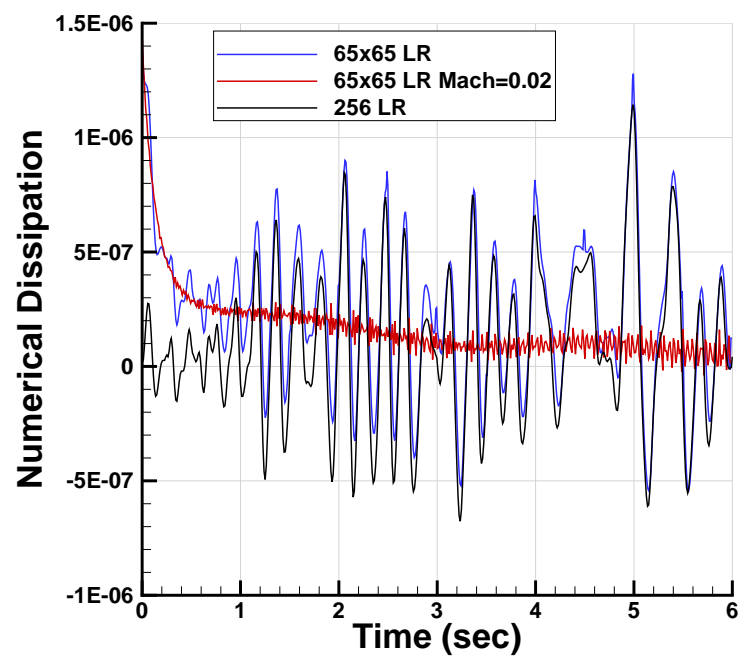

(a) Compressible Lagrange-Remap

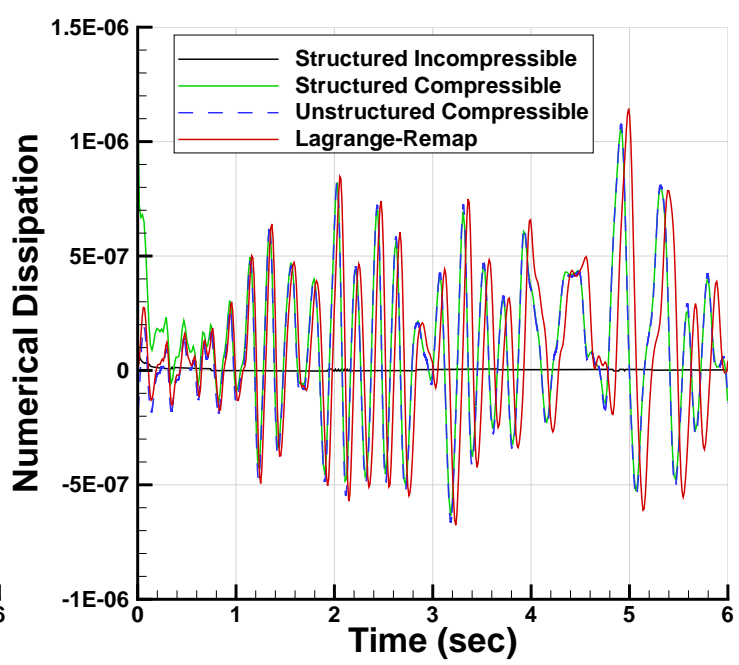

(b) Reference $256^{2}$ for all frameworks

Figure 26: Numerical dissipation for the compressible Lagrange-Remap framework on the $64 \times 64$ grid resolution as well as for all frameworks on the $256 \times 256$ reference grid resolution. The oscillations are a manifestation of acoustic fluctuations associated with compressibility effects (see Sec. 5.3 for further discussion).

\subsection{Computational Efficiency}

The evolution of momentum thickness in time (see §5.2) indicated the presence of a non-monotone converging behavior towards the end of the simulation $(t=6.0 \mathrm{sec})$. Therefore, to assess numerical efficiency $v s$. error reduction, we have chosen to measure the average percentage of the momentum thickness error from the reference solution (of each framework) during the entire simulation. The computational times are normalised with respect to a reference 
computational time, which is chosen to be the fastest simulation of each individual framework. Typically, this is the simulation time of the first-order scheme employed at grid resolution $(32 \times 32)$.

The error is calculated by:

$$
\text { Error } \%=\frac{1}{T} \int_{t=0}^{T} \frac{\left|\theta_{S}(t)-\theta_{R}(t)\right|}{\theta_{R}(t)} d t \times 100 \%
$$

where the momentum thickness $\theta(t)$ is given by equation (15). The term $\theta_{S}$ refers to the values obtained from the under-resolved simulations, while $\theta_{R}$ stands for each numerical framework reference simulation on the $256 \times 256$ grid. $T$ is the total simulation time, which is $T=6 \mathrm{sec}$ and $T=60 \mathrm{sec}$ for the Mach numbers of 0.2 and 0.02 , respectively.

In Figure 27, equation (19) is used to obtain an error estimate for each scheme on the $32^{2}$ and $64^{2}$ grids. The results are plotted against the normalized total simulation time, as detailed above, in order to highlight the efficiency of each numerical scheme as the order of accuracy increases.

Figure 27 reveals that:

1. Increasing the spatial order of accuracy reduces the numerical error; this applies to all computational frameworks employed here.

2. In the incompressible structured-grid framework, the WENO methods provided the most optimal convergence rate (Figure 27(a)).

3. For the compressible structured-grid framework (Figure 27(b)), the low-Mach number correction significantly improves the accuracy of all schemes apart from the WENO $9^{\text {th }}$ order. This indicates that for very high-order schemes and at a sufficient grid resolution, the low-Mach number correction may not provide any further benefits.

4. The error of the compressible unstructured-grid framework (Figure 27(c)) is dependent both on the mesh type and numerical scheme employed. In general, grids comprising triangular cells reduce the error faster than the corresponding quadrilateral grids.

5. The compressible Lagrange-Remap (Figure 27(d)) is the fastest of all computational frameworks employed in this study, although the slope of the error reduction as a function of computational time is not as steep as for some of the other methods.

6. The lowest errors at both grid resolutions are obtained with the compressible unstructured-grid framework. This is traded against their computational expense associated with the multidimensional nature of the reconstruction, high-order quadrature surface and volume integral, as well as with the indirect data accessing due to the unstructured grid meshes. 


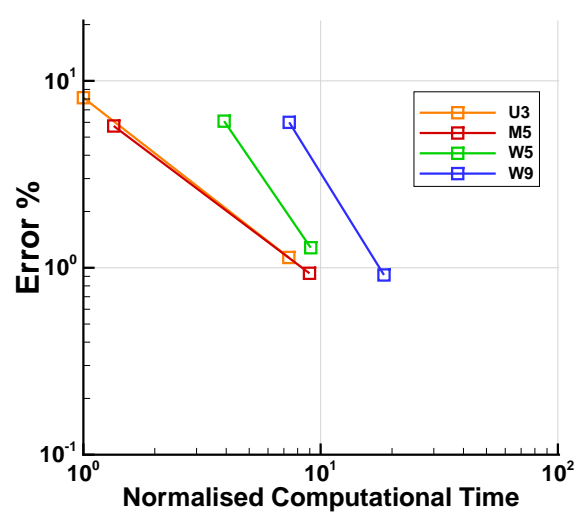

(a) Incompressible structured-grid

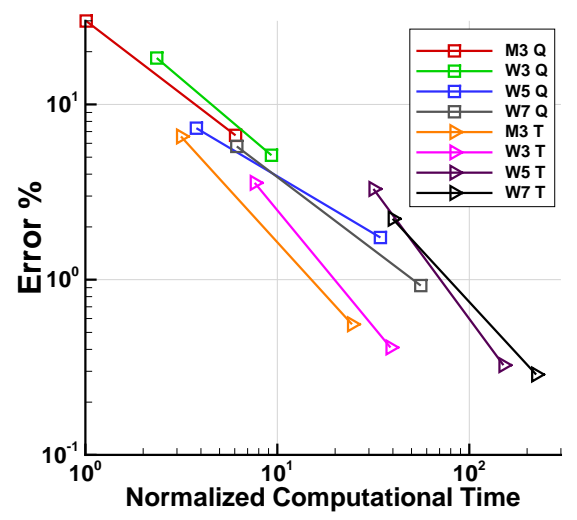

(c) Compressible unstructured-grid

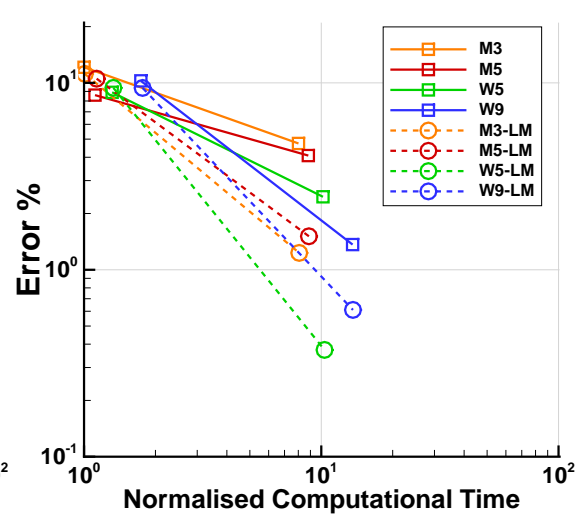

(b) Compressible structured-grid

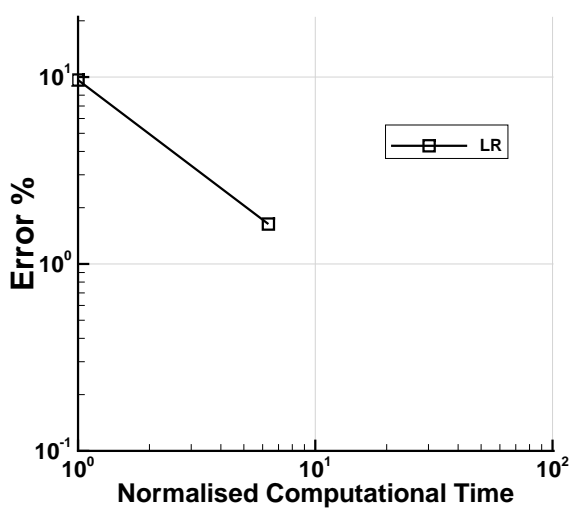

(d) Compressible Lagrange-Remap

Figure 27: Percentage of momentum thickness error during the entire simulation as a function of normalized computational time for all numerical frameworks; Point 1: $32 \times 32$, Point 2: $64 \times 64$

\section{Conclusions}

The main conclusions of the investigation of accuracy and efficiency of different computational frameworks and numerical schemes for the double-vortex pairing problem are summarised below:

1. Unstructured triangular meshes provided better results with respect to vortex evolution, momentum thickness, and dissipation rates, than their quadrilateral counterparts. It can be surmised that this is not due to the more compact nature of the associated stencils but rather on the arbitrary nature of the orientation of these elements with respect to the flow features. The exact mechanism underpinning this behavior is not fully understood, thus further investigation is required.

2. The only finite volume compressible framework that captures the correct vortex pattern and non-linear mixing growth in the low-Mach regime at the coarsest grid resolution without any low-Mach number correction or preconditioning is the unstructured framework. The WENO $5^{\text {th }}$ order scheme was found to provide sufficient 
accuracy for obtaining the correct results. This can be attributed to the fact that the compressible unstructuredgrid is the only framework for which the reconstruction is performed in characteristic space, and the $k^{\text {th }}$-order WENO schemes of this framework entails the non-linear combination of $(k-1)$-order reconstruction polynomials $[25,26]$.

3. The compressible Lagrange-Remap framework is computationally the fastest one, although the momentum thickness error does not reduce as fast as for the other numerical frameworks, especially when higher-order schemes are utilized. The compressible Lagrange-Remap in conjunction with a $3^{\text {rd }}$ order scheme can capture the correct double vortex pattern even at the lowest grid resolution,while additionally the results hardly vary with Mach number. On the other hand, the compressible structured-grid methods require at least $5^{\text {th }}$ order of accuracy to capture the same vortical structures.

4. The effect of higher order reconstruction is greater for the compressible structured-and unstructured-grid finite volume methods than the incompressible structured-grid and compressible Lagrange-Remap frameworks.

5. The low-Mach number correction has a smaller effect on the results as the order of the spatial accuracy is increased.

6. Finally, the answer given here to the question raised in the introduction "What is the lowest resolution that can be used to model a basic vortical structure?", is that at a $32 \times 32$ grid resolution the momentum thickness and large scale structures are well resolved provided that either the numerical schemes are of a high-order of accuracy or the numerical framework is sufficiently non-dissipative.

\section{Acknowledgement}

Dimitris Drikakis wishes to express his gratitude and appreciation to AWE for their financial support through the William Penney Fellowship award. The manuscript contains material @British Crown Owned Copyright 2015/MoD, reproduced with permission. Discussions with Mike Probyn and Ben Thornber are also acknowledged.

\section{References}

[1] J. Boris, F. Grinstein, E. Oran, R. Kolbe, New insights into large eddy simulation, Fluid Dynamics Research 10 (1992) 199-228, doi: 10.1016/0169-5983(92)90023-P.

[2] D. L. Youngs, Three-dimensional numerical simulation of turbulent mixing by Rayleigh-Taylor instability, Physics of Fluids A 3 (5) (1991) 1312-1320, doi:10.1063/1.858059.

[3] F. F. Grinstein, L. G. Margolin, W. J. Rider, Implicit Large Eddy Simulation: Computing Turbulent Fluid Dynamics, Cambridge University Press, ISBN 9781139463058, 2007. 
[4] D. Drikakis, Advances in turbulent flow computations using high-resolution methods, Progress in Aerospace Sciences 39 (6???7) (2003) 405-424, doi:10.1016/S0376-0421(03)00075-7.

[5] A. Mosedale, D. Drikakis, Assessment of very high order of accuracy in implicit LES models, Journal of Fluids Engineering 129 (12) (2007) 1497-1503, doi:10.1115/1.2801374.

[6] F. Grinstein, D. Drikakis, Computing turbulent flow dynamics with implicit large eddy simulation, Journal of Fluids Engineering 129 (12) (2007) 1481-1482, doi:10.1115/1.2822659.

[7] W. Rider, L. Margolin, From Numerical Analysis to implicit Subgrid Turbulence Modeling, 16th AIAA Computational Fluid Dynamics Conference doi: $10.2514 / 6.2003-4101$.

[8] J. A. Domaradzki, Z. Xiao, P. K. Smolarkiewicz, Effective eddy viscosities in implicit large eddy simulations of turbulent flows, Physics of Fluids 15 (12) (2003) 3890-3893, doi:10.1063/1.1624610.

[9] K. Ishiko, N. Ohnishi, K. Ueno, K. Sawada, Implicit Large Eddy Simulation of Two-Dimensional Homogeneous Turbulence Using Weighted Compact Nonlinear Scheme, Journal of Fluids Engineering 131 (6) (2009) 061401-0614014, doi:10.1115/1.3077141.

[10] C. D. Winant, F. K. Browand, Vortex pairing : the mechanism of turbulent mixing-layer growth at moderate Reynolds number, Journal of Fluid Mechanics 63 (1974) 237-255, doi:10.1017/S0022112074001121.

[11] S. Shanmuganathan, D. L. Youngs, J. Griffond, B. Thornber, R. J. R. Williams, Accuracy of high order density based compressible methods in low Mach vortical flows, International Journal for Numerical Methods in Fluids 74 (2014) 335-358, ISSN 1097-0363, doi:10.1002/fld.3853.

[12] P. Drazin, W. Reid, Hydrodynamic Stability, Cambridge University Press, 2004.

[13] S. K. Godunov, Finite difference method for numerical computation of discontinuous solutions of the equations of fluid dynamics, Matematicheski Sbornik 47 (1959) 271-306.

[14] D. Drikakis, J. Rider, High Resolution Methods for Incompressible and Low-Speed Flows, vol. 1, Springer-Verlag, Heidelberg, 2004.

[15] E. F. Toro, Riemann Solvers and Numerical Methods for Fluid Dynamics - A Practical Introduction, Berlin: Springer-Verlag, 1997.

[16] B. van Leer, Towards the ultimate conservative difference scheme. II. Monotonicity and conservation combined in a second-order scheme, Journal of Computational Physics 14 (4) (1974) 361-370, ISSN 0021-9991, doi:10.1016/0021-9991(74)90019-9.

[17] X.-D. Liu, S. Osher, T. Chan, Weighted Essentially Non-oscillatory Schemes, Journal of Computational Physics 115 (1) (1994) 200-212, ISSN 0021-9991, doi:10.1006/jcph.1994.1187.

[18] K. H. Kim, C. Kim, Accurate, efficient and monotonic numerical methods for multi-dimensional compressible flows: Part I: Spatial discretization, Journal of Computational Physics 208 (2) (2005) 527-569, ISSN 0021-9991, doi:10.1016/j.jcp.2005.02.021.

[19] K. H. Kim, C. Kim, Accurate, efficient and monotonic numerical methods for multi-dimensional compressible flows: Part II: Multidimensional limiting process, Journal of Computational Physics 208 (2) (2005) 570-615, ISSN 0021-9991, doi:10.1016/j.jcp.2005.02.022.

[20] G.-S. Jiang, C.-W. Shu, Efficient implementation of weighted ENO schemes, Journal of Computational Physics 126 (1) (1996) 202-228, doi:10.1006/jcph.1996.0130.

[21] D. S. Balsara, C.-W. Shu, Monotonicity preserving weighted essentially non-oscillatory schemes with increasingly high order of accuracy, Journal of Computational Physics 160 (2) (2000) 405-452, doi:10.1006/jcph.2000.6443.

[22] E. M. Taylor, M. Wu, M. P. Martn, Optimization of nonlinear error for weighted essentially non-oscillatory methods in direct numerical simulations of compressible turbulence, Journal of Computational Physics 223 (1) (2007) 384 - 397, ISSN 0021-9991, doi:10.1016/j.jcp. 2006.09.010.

[23] B. Thornber, A. Mosedale, D. Drikakis, D. Youngs, R. Williams, An improved reconstruction method for compressible flows with low Mach number features, Journal of Computational Physics 227 (10) (2008) 4873-4894, ISSN 0021-9991, doi:10.1016/j.jcp.2008.01.036.

[24] C.-W. Shu, S. Osher, Efficient Implementation of Essentially Non-Oscillatory Shock-Capturing Schemes, Journal of Computational Physics 
77 (2) (1988) 439-471, ISSN 0021-9991, doi:10.1016/0021-9991(88)90177-5.

[25] V. Titarev, P. Tsoutsanis, D. Drikakis, WENO schemes for mixed-element unstructured meshes, Communications in Computational Physics 8 (3) (2010) 585-609, doi:10.4208/cicp.040909.080110a.

[26] P. Tsoutsanis, V. Titarev, D. Drikakis, WENO schemes on arbitrary mixed-element unstructured meshes in three space dimensions, Journal of Computational Physics 230 (4) (2011) 1585-1601, ISSN 0021-9991, doi:10.1016/j.jcp.2010.11.023.

[27] A. Antoniadis, P. Tsoutsanis, D. Drikakis, High-order schemes on mixed-element unstructured grids for aerodynamic flows, 42nd AIAA Fluid Dynamics Conference and Exhibit 2012 .

[28] P. Tsoutsanis, A. F. Antoniadis, D. Drikakis, WENO schemes on arbitrary unstructured meshes for laminar, transitional and turbulent flows, Journal of Computational Physics 256 (0) (2014) 254-276, ISSN 0021-9991, doi:10.1016/j.jcp.2013.09.002.

[29] A. Haselbacher, A WENO reconstruction algorithm for unstructured grids based on explicit stencil construction, 43rd AIAA Aerospace Sciences Meeting and Exhibit - Meeting Papers (2005) 3369-3378.

[30] G. Hu, R. Li, T. Tang, A robust WENO type finite volume solver for steady Euler equations on unstructured grids, Communications in Computational Physics 9 (3) (2011) 627-648, doi:10.4208/cicp.031109.080410s.

[31] M. Dumbser, M. Käser, Arbitrary high order non-oscillatory finite volume schemes on unstructured meshes for linear hyperbolic systems, Journal of Computational Physics 221 (2) (2007) 693-723, ISSN 0021-9991, doi:10.1016/j.jcp.2006.06.043.

[32] M. Dumbser, W. Boscheri, High-order unstructured Lagrangian one-step WENO finite volume schemes for non-conservative hyperbolic systems: Applications to compressible multi-phase flows, Computers \& Fluids 86 (2013) 405-432, doi:10.1016/j.compfluid.2013.07.024.

[33] T. Barth, D. Jespersen, The design and application of upwind schemes on unstructured meshes, 27th AIAA Aerospace Sciences Meeting and Exhibit .

[34] V. Garanzha, L. Kudryavtseva, S. Utyuzhnikov, Variational method for untangling and optimization of spatial meshes, Journal of Computational and Applied Mathematics 269 (2014) 24-41.

[35] V. Titarev, E. Toro, ADER: Arbitrary High Order Godunov Approach, Journal of Scientific Computing 17 (1-4) (2002) 609-618.

[36] B. van Leer, Towards the ultimate conservative difference scheme. V. A second-order sequel to Godunov's method, Journal of Computational Physics 32 (1) (1979) 101-136, ISSN 0021-9991, doi:10.1016/0021-9991(79)90145-1.

[37] B. van Leer, Towards the ultimate conservative difference scheme. IV. A new approach to numerical convection, Journal of Computational Physics 23 (3) (1977) 276-299, ISSN 0021-9991, doi:10.1016/0021-9991(77)90095-X.

[38] O. A. Ladyzhenskaya, Mathematical Problems in the Dynamics of a Viscous Incompressible Flow, Gordon and Breach, New York, 1963.

[39] A. J. Chorin, Numerical Solution of the Navier-Stokes Equations, Mathematics Of Computation 22 (104) (1968) 745-762, doi:10.1090/ S0025-5718-1968-0242392-2.

[40] R. Temam, Sur l'approximation de la solution des équations de Navier-Stokes par la méthode des pas fractionnaires (I), Archive for Rational Mechanics and Analysis 32 (2) (1969) 135-153, ISSN 0003-9527, doi:10.1007/BF00247678.

[41] A. J. Chorin, A Numerical Method for Solving Incompressible Viscous Flow Problems, Journal of Computational Physics 2 (1) (1967) 12-26, ISSN 0021-9991, doi:10.1016/0021-9991(67)90037-X.

[42] L. Könözsy, D. Drikakis, A unified fractional-step, artificial compressibility and pressure-projection formulation for solving the incompressible Navier-Stokes equations, Communications in Computational Physics 16 (5) (2014) 1135-1180, doi:10.4208/cicp.240713.080514a.

[43] L. Könözsy, Multiphysics CFD Modelling of Incompressible Flows at Low and Moderate Reynolds Numbers, Ph.D. thesis, Cranfield University, College of Aeronautics, Department of Engineering Physics, URL https://dspace.lib.cranfield.ac.uk/bitstream/1826/ 7860/1/Laszlo_Konozsy_Thesis_2012.pdf, 2012.

[44] D. Drikakis, P. A. Govatsos, D. E. Papantonis, A characteristic-based method for incompressible flows, International Journal for Numerical 
Methods in Fluids 19 (8) (1994) 667-685, ISSN 1097-0363, doi:10.1002/fld.1650190803.

[45] E. Shapiro, D. Drikakis, Artificial compressibility, characteristics-based schemes for variable density, incompressible, multi-species flows. Part I. Derivation of different formulations and constant density limit, Journal of Computational Physics 210 (2) (2005) 584-607, ISSN 0021-9991, doi:10.1016/j.jcp.2005.05.001.

[46] E. Shapiro, D. Drikakis, Artificial compressibility, characteristics-based schemes for variable-density, incompressible, multispecies flows: Part II. Multigrid implementation and numerical tests, Journal of Computational Physics 210 (2) (2005) 608-631, ISSN 0021-9991, doi: 10.1016/j.jcp.2005.05.002.

[47] J. Perot, An Analysis of the Fractional Step Method, Journal of Computational Physics 108 (1) (1993) 51-58, doi:10.1006/jcph.1993.1162.

[48] A. Quarteroni, R. Sacco, F. Saleri, Numerical Mathematics, Springer-Verlag, New York, 2000.

[49] M. Griebel, T. Dornseifer, T. Neunhoeffer, Numerical Simulation in Fluid Dynamics, Society for Industrial and Applied Mathematics (SIAM), 1998.

[50] I. Kokkinakis, D. Drikakis, Implicit Large Eddy Simulation of weakly-compressible turbulent channel flow, Computer Methods in Applied Mechanics and Engineering 287 (0) (2015) 229 - 261, ISSN 0045-7825, doi:10.1016/j.cma.2015.01.016. 